\title{
Optical Properties of Metals by Spectroscopic Ellipsometry*
}

$\Longrightarrow$

E. T. Arakawa, T. Inagaki, ${ }^{+}$and M. W. Williams

Health and Safety Research Division

Oak Ridge National Laboratory

Oak Ridge", Tennessee 37830

\footnotetext{
By acceptance of this article, the

publisher or recipient acknowledess

the U.S. Government's ripht to

retein a nonexclusive, royelty-free

licence in end to eny copyrifht

covering the article.
}

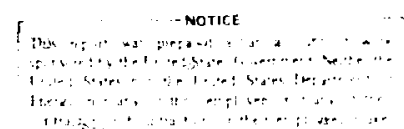

* Research sponsored by the Office of Health and Environmental Research, U. S. Department of Energy, under contract H-7405-eng-26 with the Union Carbide Corporation.

Present address: Department of Physics, Osaka Kyoiku University, Tennoji, Osaka, Japan. 


\section{Optical Properties of Metals by Sper.troscopic Ellipsometry.}

E. T. Arakawa, T. Inagaki,* and M. W. Williams

Health and Safety Research Division, Oak Ridge National Laboratory, Oak Ridge, Tennessee 37830

\section{Abstract}

The use of spectroscopic ellipsometry for the accurate determination of the optical properties of liquid and solid metals is discussed and illustrated with previously published data for $L i$ and Na. New data or liquid $\mathrm{Sn}$ and $\mathrm{Hg}$ from 0.6 to $3.7 \mathrm{eV}$ are presented. Liquid $\mathrm{Sn}$ is Drudelike. The optical properties of tig deviate from the Drude expressions, but sinultaneous measurements of reflectance and ellipsonetric parameters yield consistent results with no evidence for vectorial surface effects.

\section{MASTER}

*Present address: Department of Physics, Osaka Kyoiku University, Tennoji, Osaka, Japan. 


\section{Introduction}

For many years ellipsonetry has been a widely used technique in the study of surfaces. It has long been recognized, in theory, that ellipsometry is capable of yielding highly accurate values of the bulk optical properties for a clean sample as well as being serisitive to very thin surface filns or to small amounts of adsorbed materials. Before the advent of ultrahigh vacuun techniques, it was difficult to produce and maintain sufficiently clean surface; for the deternination of accurate values of the bulk opticai properties of a metal by ellipsometry. Because of this and the known sensitivity of ellipsonetry to surface films, one of its more routine applications has been to monitor a change occurring at a surface with time (e.g., $3 x i d a t i o n$ of the surface), as long as this did not proceed too rapidly. A single ellipsometric measurement was quite complex and time consuming, and extraction of the optical properties of the sample from the measured ellipsonetric paranieters was computationally challenging. For these reasons early ellipsometers were mostly designed for use at a single wavelength. With the development of fast automated ellipsometers and the almost universal availability of adequate computing facilities, spectroscopic ellipsonetry has now become a very powerful tool, with enormous potential, for the study of metals [1-5]. Combined with present day ultrahigh vacuum teciniques, spectroscopic ellipsometry can now yield accurate bulk optical prorrties of clean samples. In addition, simultaneous ellipsometric al eflectance measurements on the same clean sample can yield informe on about intrinsic properties of the surface. The application of ellipsometry to the study of thin surface films is currently expanding in scope. It is anticipated, for example, that there 
will be increased use of ellipsometry in many biological and medical problems involving membranes or macromolecules on surfaces $[6,7]$. Interferometric ellipsometry [8], ellipsometry combined with surface e]ectromagnetic waves [9], and the study of multiiayer systens by a combination of internal and external ellipsometry [10] will all, undoubtedly, be expanded to a range of wavelengths. These spectroscopic ellipsometry techniques will each yield specific information about the surface that is probed. Different types of ellipsometric systems with their advantages and disadvantages and the accuracies attainable have been reviewed by Aspnes [1] and by Kinosita and Yamarioto [11].

In this review we wili demonstrate the necessity of employing spectroscopic ellipsometry techriques in the determination of accurate values for the optical properties of solid and liquid metals in the energy range from the infrared to the near ultraviolet. This energy range, below the volume plasmon energy for most metals, is an energy range of great interest. However, the optical conductivity is usually very high over much of this region, and thus the normal incidence reflectance is relatively insensitive to the optical properties of the metal. Reflection ellipsometry is far more sensitive, both to the optical properties of the bulk metal and to the surface conditions. Because of this sensitivity to surface conditions, early data obtained by spectroscopic ellipsometry for a particular material were not necessarily reproducible from one experimental arrangement to the next. One example is the so-called Mayer-El Naby anomaly in the optical absorption of alkali metals, first reported in 1963. It is only recentiy that consistent, accurate data characteristic of the clearl metal have been obtained using ultrahigh vacuum reflection ellipsonetry. 
Optical properties have been obtained for most metals in their solid state over an extended energy range. In Section I we discuss recent studies of the optical properties of solid $\mathrm{Li}$ and $\mathrm{Na}$, with emphasis on the accuracy attainable. Optical properties have been obtained for relatively few liquid metals and, in general, these are over very limited energy ranges. Existing data for liquid metals are discussed, and new data on Tiquid Sn are presented in Section II. In Section III we present new data on liquid $\mathrm{Hg}$. The optical properties of liquid $\mathrm{Hg}$ have evoked particulär interest. A consistent difference has been observed between the optical properties deduced from reflectance and from ell ipsometric spectra, which has led to the proposal of various surface models. We show that no real differences are found between the optical properties deduced from reflectance and from ellipsometric measurements and that there is no evidence for any surface structure for liquid $\mathrm{Hg}$. The optical constants of bulk Hg, obtained most accirately by ellipsometry, are, however, nonDrude-like and this deviation from classical behavior requires theoretical explanation. 


\section{Solid Li and $\mathrm{Na}$}

For many years interest in the alkali metals centered around anomalous structure seen by some workers in the IR to visible region of the absorption spectra.| As an example, most of the experimental results for solid Na, published up to 1974, are shown in Fig. 1 in the form of the optical conductivity o defined by $\sigma=\omega \varepsilon_{2} / 4 \pi$, where $\varepsilon_{2}$ is the imaginary part of the dielectric function and $\omega$ is the angular frequency of the incident photons. All of these results were obtained using ellipsometric techniques. It was realized that, for the alkali metals in this energy region, the high values observed for reflectance make it difficult to obtain accurate values of the optical properties. This also applies for other solid and liquid metals and will be demonstrated for $\mathrm{Hg}$ in Section III. From the data shown in Fig. I for solid $\mathrm{Na}$, only Smith's results [12] can be interpreted in terms of a nearly-free-electron theory and the known band structure of $\mathrm{Na}$. The doublepeak structure observed by Mayer and Hietel [13] cannot be explained by a simple theory and was termed anomalous. It is to be noted that even studies performed after the development of ultrahigh vacuum techniques show significantly different spectra, presumably due to such factors as the roughness, crystallinity, granularity, and contamination of the surfaces studied.

As part of a program designed to study the optical properties of liquid alkali metals, we determined the optical properties of $\mathrm{Na}$ and $\mathrm{Li}$, between 0.6 and $3.8 \mathrm{eV}$, in the form of thick evaporated solid films [14]. The ellipsometer and experimental techniques have been described previously [14]. The samples were prepared and measured under ultrahigh vacuum conditions: 
before and after evaporation, the pressure in the sample compartment was in the low $10^{-9}$ torr range. Measurements were made at room temperature by reflection ellipsometry through a quartz substrate at the quartz-metal interface. Our results [14] for solid $\mathrm{Na}$, compared with those of Smith [12], are shown in Fig. 2. The anomalous structure reported by Mayer and Hietel was not seen: rather Smith's results were confirmed for the first time.

Among the wide variety of ellipsometric techniques, the photorietric method of Conn and Eaton [15] was employed in this study to measure $\psi$ and $\Delta$ defined by $\tan \psi=r_{p} / r_{s}$ and $\Delta=\Delta_{p}-\Delta_{s}$, respectively, where $r_{p}$ and $r_{s}$ are the reflectance amplitudes, and $\Delta_{p}$ and $\Delta_{s}$ are the phase changes upon reflection, of the conponents of the incident light with the electric vector parallel and perpendicular to the plare of incidence, respectively. In this method, by setting the polarizer at $45^{\circ}, \psi$ is determined from the intensity ratio $I_{p} / I_{s}$ of the $p$ and $s$ components of reflected light by $\tan \psi=\left(I_{p} / I_{s}\right)^{\frac{1}{2}}$, and $\Delta$ is determined from the axial ratio $\left(I_{\min } / I_{\max }\right)^{\frac{1}{2}}$ of the ellipse of the reflected 1 ight by $\sin \Delta=\sin 2 \xi / \sin 2 \psi$, where $I_{\min }$ and $I_{\max }$ are the minimum and maximum intensities measured by rotating the analyzer around the axis of the reflected beam.and $\tan \xi=\left(I_{\min } / I_{\max }\right)^{\frac{1}{2}}$.

The advantage of this method is its high sensitivity in determining $\psi$. As has been discussed by Smith [12], if $\beta$ is defined by $\psi=\frac{1}{4 \pi}-\beta, \beta$ is quite small for a highly reflecting surface for which $r_{p} / r_{s}$ is very close to unity. In fact, using the conventional null method, in which the directly measured quantity is essentially the angle $\psi$, Smith [12] could not obtain reliable values of $\psi$ from a single reflection at a 
quartz-Na interface due to the relatively low sensitivity of the nuil method. In contrast, since the intensity ratio $I_{p} / I_{s}$ can be written for small values of $\beta$ as $I_{p} / I_{s}=\tan ^{2} \psi \simeq 1-4 \beta$, the present method is four times more sensitive than the null method. This method has even higher sensitivity than the modulation method developed by Jasperson and Schnacterly [16] in which tise quantity measured to determine $\psi$ is $\left(I_{s}-I_{p}\right) /\left(I_{s}+I_{p}\right) \simeq 2 \beta$. The contrast between the present method and the null method can be seen clearly in Fig. 3. The values of $\beta$ measured by Smith [12] for a single reflection at a quartz-Na interface at an angle of incidence of $75^{\circ}$ are plotted using open circles in the lower part of Fig. 3. Since no particular structure was found in these data, he had to make multiple reflection measurements, in which he actually measured the quantities $4 \beta$ and $7 \beta$ from four and seven reflections at quartz-Na interfaces. His data for $4 \beta$ are shown in the upper part of Fig. 3 by open circles and these do exhibit a clear structure. The present data obtained from a single reflection at a $70^{\circ}$ angle of incidence, and presented by filled circles in the figure, show a strusture similar to that in Smith's $4 \beta$ data. A technique similar to the present one has been used in Mayer and Hietel's study [13] on $\mathrm{Na}$ and in a study of solid Li by Mathewson and Myers [17].

As had been found previously by Smith [12] the experimental values of the optical conductivity of solid $\mathrm{Na}$ below the interband threshold are considerably larger than the Drude values given by $\sigma_{D}=\mathrm{Ne}^{2} / \mathrm{m}^{*} \omega^{2} \tau$, if $\sigma_{D}$ is evaluated from the $m^{\star}$ deduced from the experimental $\varepsilon_{T}$ values, where 
$\varepsilon_{1}$ is the real part of the dielectric function, and $\tau$ from the dc conductivity. In this expression, $N$ is the density of free carriers, $m^{*}$ is their effective mass, and $\tau$ is the relaxation time. Yalues of $\varepsilon_{7}$, obtained by us, for solid $\mathrm{Na}$ are plotted in Fig. 4 as a function of $\lambda^{2}$, the square of the wavelength in $\mu \mathrm{m}$. Assuming the simple expression

$$
\varepsilon_{1}=1+4 \pi N_{0} \alpha-\left(\frac{\omega_{a}}{\omega}\right)^{2}
$$

to hold for $\varepsilon_{7}$, where $N_{0}$ is the number of atoms per unit volume, $\alpha$ is the ion core polarizability, and $\omega_{a}=\left(4 \pi \mathrm{Ne}^{2} / \mathrm{m}^{*}\right)^{\frac{1}{2}}$, the values of $\mathrm{m}^{*} / \mathrm{m}$ obtained are 1.07 in the IR and 1.08 in the visible and $u$ traviolet. The plasmon energy, $\hbar_{w_{p}}$, defined by $\varepsilon_{1}=0$ is $5.6 \mathrm{eV}$ and $4 \pi N_{0} \alpha$, obtained for $\lambda^{2}=0$, is 0.1 .

The available measurements of the room temperature optical conductivity for solid $\mathrm{Li}$ covering the energy range from the infrared to the near ultraviolet are presented in Fig. 5. The data by Inagaki et al. [14] were obtained using the same techniques as described for solid $\mathrm{Na}$. Samples were deposited and measured at room temperature, with observations made at the quartz-1jthium interface. Curve $A$ by Mathewson and Myers [17] was measured for a Li film on a quartz substrate twelve hours after deposition, curve 3 was measured for the same film seven days after deposition, and curve $C$ was obtained for a $L i$ fiim on a sapphire substrate. In each case, Li was deposited on substrates cooled by liquid nitrogen and then measurements made at room temperature at the vacuum-Li interface. Hunderi's measurements [18] were made at room temperature at the vacuum-Li interface 
on films which had been prepared by evaporation onto sapphire substrates cooled to $80^{\circ} \mathrm{K}$. Myers and Sixtensson's results [19] were obtained from measurements made at room temperature at the vacuum- $L i$ interface. The film believed to be characteristic of the BCC structure was deposited on a sapphire substrate cooled to $15^{\circ} \mathrm{K}$, while that believed to be characteristic of the HCP structure was deposited on a sapphire substrate cooled to $80^{\circ} \mathrm{K}$. Both samples were annealed at room temperature prior to measurements being made. Mathewson and Myers [17] stated that, of their data, curve $C$ was most likely characteristic of the BCC structure in Li. They further assumed that changes with time indicated a change in crystalline structure of their sample. Most of the Li fi]ms studied by Inagaki et al. [14] were stable and did not change with time. For the few films that did change, increased absorption was seen in the vicinity of $2 \mathrm{eV}$, rather than the types of change in structure seen by others and indicated by Fig. 5 .

It is known that $L i$ is a difficult metal to work with. It is highly reactive and its crystalline structure is found to depend critically on the method of film preparation. As seen in Fig. 5, there is no demonstrated consistency in the experimental optical conductivity spectra obtained from ellipsometric measurements at room temperature. Assuming the threshold for interband transitions is given by the energy corresponding to the minimum in the conductivity spectrum, none of the data shown in Fig. 5 are consistent with simple band-structure calculations. Experimentally the minima in the different studies lie in the energy range from 1.4 to $2.3 \mathrm{eV}$, whereas the predicted value is $\sim 3.0 \mathrm{eV}$. Possibly, broadening of the absorption edge may shift the apparent onset of absorption so 
that the observed minimum for a given curve should not be interpreted in this way. However, at this time the data on Li cannot be explained in terms of simple theories based on the nearly-free-electron model of a metal.

Rasigni and Rasigni [20] have presented optical constants for $\mathrm{Li}$ between 0 and $10.7 \mathrm{eV}$, calculated by a Kramers-Kronig analysis of normal incidence reflectance data. The reflectance values between 0.5 and $5 \mathrm{eV}$ were obtained by direct measurement on a vacuum-Li interface at $6^{\circ} \mathrm{K}$, while from 5.5 to $10.7 \mathrm{eV}$ they were calculated from the optical constants determined, at room temperature, by Callcott and Arakawa [21] from observations of reflectance as a function of angle of incidence at both the vacuum-Li and substrate-Li interfaces. Their Kramers-Kronig analysis was normalized to Inagaki et a]. [14] from 2 to $3 \mathrm{eV}$ and to Cal1cott and Arakawa [21] from 6.5 to $? \mathrm{eV}$. Values of the refractive index, $\mathrm{n}$, and extinction coefficient, $k$, obtained by Rasigni and Rasigni are shown in Fig. 6 compared with the same quantities obtained by Inagaki et al. and Callcott and Arakawa. It is seen that, even with the normalization of the KramersKronig analysis in the 2 to $3 \mathrm{eV}$ region, there are significant differences between the optical constants obtained from reflectance and from ellipsometric measurements in the infrared and visible regions.

$L i$, with its low atomic number and relatively simple atomic structure, is of great theoretical interest. Further experimental studies of the optical properties of solid, and liquid, Li under controlled conditions, coupled with surface characterizations, are required. 


\section{Liquid Metals}

Liquid metal samples in equilibrium with their environment are free of macroscopic surface roughness effects. In addition, geometrical factors involving granularity and different crystalline forms of the metal which can affect the values obtained for the optical properties of solid metals are not encountered for liquid metals. Under ultrahigh vacuum conditions, the surface can also be obtained free of contamination. Thus, in principle, it should be easier to obtain accurate values of the bulk optical properties of a metal in its liquid state than in its solid state. Experimentally, the temperatures required to melt most metals make optical measurements under ultrahigh vacuum conditions difficult to perform. Consequently there is a relative sparsity of optical data on liquid metals other than $\mathrm{Hg}$.

Existing optical data on liquid metals obtained by ellipsometric techniques are mainly attributable to Kent [22] ( $\mathrm{Cd}, \mathrm{Sn}, \mathrm{Pb}, \mathrm{Bi}$ ), Hodgson [23]. ( $\mathrm{Cu}, \mathrm{Ge}, \mathrm{Ag}, \mathrm{Cd}, \mathrm{In}, \mathrm{Sn}, \mathrm{Sb}, \mathrm{Te}, \mathrm{Hg}, \mathrm{Pb}, \mathrm{Bi}$ ), Lelyuk' et a1. [24] (Ga, $\mathrm{Hg}$ ), Mayer and Hietel [13] (Na, K, Rb, Cs), Smith [25j (Cd, Hg, Pb, Bi), Miller [26] (A1, $\mathrm{Fe}, \mathrm{Co}, \mathrm{Ni}, \mathrm{Cu}, \mathrm{Ag}, \mathrm{Au})$, Comins [27] (A], Cu, Ga, Sn, $\mathrm{Au}, \mathrm{Hg}, \mathrm{Pb}, \mathrm{Bj}$ ), and Inagaki et ai. [28] (Na). Measurements of reflectance have been employed by Schulz [29] (Ga, $\mathrm{Hg})$; and Wilson and Rice [30] ( $\mathrm{In}, \mathrm{Hg}, \mathrm{Bi}$ ). In addition there are several other determinations of the optical properties of liquid $\mathrm{Hg}$ which will be referenced in Section III. Many of these measurements on liquid metals are over a very limited energy range. Exceptions are the reflectance spectra obtained by Wilson and Rice from 2 to $20 \mathrm{eV}$. It has been found that the optical properties 


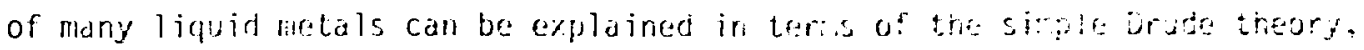
at least over limited energy ranges, as long as mor for "an effective density of frese carriers) and a are treated as adjustabif paracters, i.e., the optical properties of many of the liquid metais mich have testen studirad

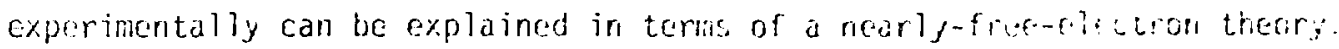
A notable exception is liquid Hg, which will be discussed in section lll. In fact, simple metals, having weak electron-ion interactions (e.f., iaj, Al, etc.) are described by a nearly-free-electron theory and car, be expected to obey the orude formulae, whereas for hetals where the seatterific interaction is strong (e.g., transition and rare earth net:als, divalent metals, Be, Hg, Ba, etc.) there are more uncertainties in the theory and more probability of non-Drude-1ike behavior [31]. In addition, band gaps characteristic of long-range order are destroyed by melting, and it is to be expected that the corresponding structures would be removed from the energy spectrum of the liquid metal $[31,32]$. For several meta]s. structures in the optical properties of the solid associated with transitions from the conduction band to states above the Fermi level have not been observed in the spectrum for the liquid. On the other hand, we will show that structure for 1 iquid $\mathrm{Na}$ appears at the same energy as structure for solid $\mathrm{Na}$. When transitions in the solid metal are from the d-bands to the Fermi level, structure has been found to persist in the spectrum for the liquid $[26,30]$. Thus, as might be expected, some short-range order exists in liquid metals which is similar to that which exists in the corresponding solid. In fact, from theoretical and experimental arguments, based principally on correlations between nuclear magnetic resonance nieasureilents and electronic structure, Knight and Berger [33] concluded that a liquid metal possesses a band structure which is very like that of its solid, 


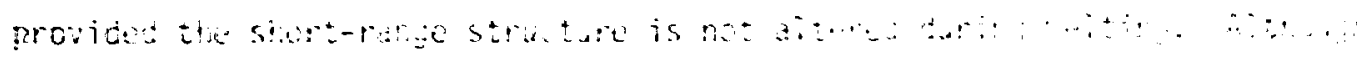

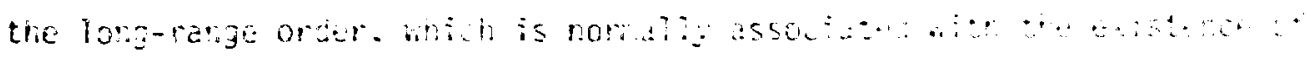

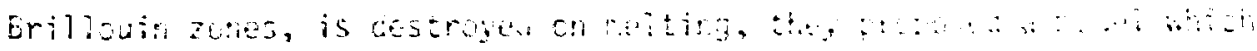

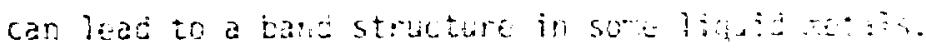

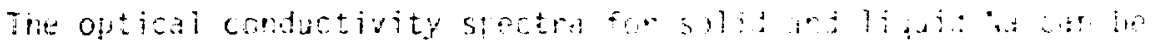

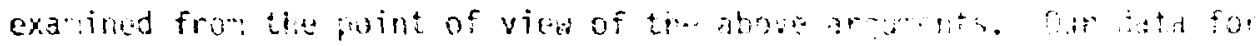

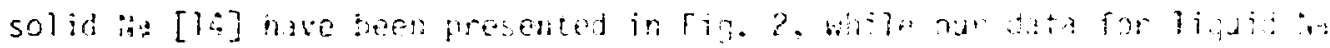

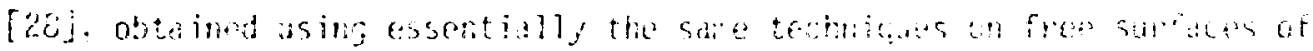
ila at $120 \mathrm{C}$, are shom in 7 il. 7 . In contras to the case of solit ita,

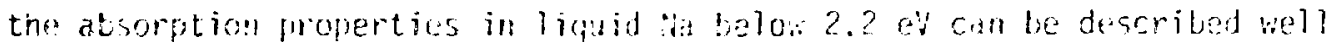
by tire simple Brode model of Prec-carrier absorption. Ahnur.? el, absorition is greater than predicted by the simpie prude model. Although Saiti: [3a] has shown throretically that the interband transition in solid fia, seen in the region of 3 el in Fig. 2, should not persist into the liguid phase, knight and Berger [33] suggest, from observations of nuclear magnetic resonance line shifts in liquid and solid alkali metals, that the electronic structures of the respective solids and liquids are the same. This bould justify our interpretation [23] of the structure seen in the optical conductivity of liquid sodium above about $2.2 \mathrm{eV}$ as being due co a liquid analog of the interband absorption seen in the spectrum for the solid. On the other hand, Helman and Baltensperger [35] clain that this discrepancy between our experinental results and the simple Drude formula does not arise from an interband transition but from the fact that the relaxation time $i$ is frequency dependent when the electron-ion interaction in the liquid metal is property taken into account. 
We have rosestly obtained the optical properties of liquid $5 n$ [36] at $26]^{\circ} \mathrm{C}$ frow ellipsometric measurements for photon energies between 0.62 and 3.7 eV. Measurements were made at a vacuum-1 iquid $S n$ interface after contaninants were renoved from the liquid surface using a stainless steel scraper. The real and ilmaginary parts, $\varepsilon_{7}$ and ' 2 , of the dielectric function are shown in Fig. 8 together with the values obtained previously by kent [22], Hodgson [23] ard Comins [27], and the corresponding Drude curves. Figure 9 shows the variation of $\left(1-q^{-1}\right.$ with $\lambda^{-2}$ for our values of 1 for liquid Sn. Tlis curve is of interest since all data from the infrared through the ultraviolet fall on a single straight line yielding a single value of $m^{*}$ from the slope. This is coricrary to the results for ather metals in the solid and liquid phases (compare Fig. 4) where different values of r.* $^{*}$ are obtained in the infrared and in the visible and ultraviolet regions. It is seen that liquid $S n$ behaves like a nearly-free-electron metal over the full range of the experimental measurements. There is no evidence of the interband transitions seen in solid $5 \mathrm{n}$ at $1.2 \mathrm{eV}$ and $\sim 3$ eV [37]. A value of $\mathrm{In}^{*} / \mathrm{m}=0.98 \div 0.05$ was obtained fron the slope of Fig. 9, compared with 23.0 for solid Sn [36]. The sum rule

$$
\int_{0}^{\infty} \sigma(1) d \omega=\frac{\pi}{2}\left(\frac{N_{T}}{m}\right) e^{2},
$$

where $N_{T}$ is the total electron density, requires that if some interband transitions of various electrons disappear on melting, then the value of $m^{*}$ representing intraband transitions will decrease. This is true for Sn $[32]$. 
The measurenent of the optical properties of iiquid lietals over an extended energy range, from the infrared to the vacuum u? traviolet, deserves more attention. In particular, the observation of the optical properties of a metal in both the solid and liquid states obtained in the sane experiment under ultrahigh vacuum conditions by spectroscopic ellipsometry would be of great interest. Accurate measurements, on well-characterized surfaces, would yield information of relevance to theories of order-disorder on going from the solid to the liquid [38]. 


\section{I11. Liquid Hg}

Mercury would appear, at first, to be an easy place to start in a study of the optical properties of liquid metals. It is easy to handle, stable, and liquid at room temperature. Furthermore, it is relatively easy to obtain a clean $\mathrm{Hg}$ surface. In 1957, Schulz [29] surveyed the literature and on the basis that there were many measurements available in the visible, some in the ultraviolet, and a few in the infrared for liquid $\mathrm{Hg}$, and only the measurements by Kent [22] at three wavelengths in the visible available for other liquid metals, came to the conclusion that "If the goal is to find a metal which is most likely to follow the Drude theory over a long wavelength range, the evidence points toward liquid Hg as being the most promising metal." Schulz, from his reflectance measurements, then presented a strong case for the validity of the Drude theory in liquid $\mathrm{Hg}$. Since then a consistent difference has been observed between the optical properties deduced from reflectance and from ellipsometric spectra. To reconcile the two sets of results, bioch and Rice [39] proposed a surface transition zone. From ellipsometric and reflectance measurements made simultaneously on the same samples, Crozier and Murphy [40] concluded that such a model, coupled with vectorial optical properties for the liquid $\mathrm{Hg}$, was consistent with their data. Guidotti and Rice [41] probed the liquid $\mathrm{Hg}$ surface directly using an attenuated total reflection method to excite surface plasmons and expressed the vectoria? effect in terms of a conductivity in the surface layer which is both anisotropic and larger than the value in the bulk. We have recently measured simultaneously the absolute reflectances $R_{s}$ and $R_{p}$ and the 
ellipsonetric parameter $\Delta$ for liquid $\mathrm{Hg}$ over the energy range from 0.6 to $3.7 \mathrm{eV}$ [42]. No systematic differences were found between the optical properties deduced from reflectance and from ellipsometry, in contrast to the results of Crozier and Murphy. In this Section we present an analysis to demonstrate that neither the previously existing nor our new ellipsometric data show evidence for any intrinsic surface structure in liquid Hg. By use of a kramers-kronig analysis it is then shown that it is the optical properties obtained from ellipsometric measurements that are representative of 1 iquid $\mathrm{Hg}$. Finally, it is shown that there is no real conflict with the optical properties deduced from reflectance data as there are large inherent uncertainties associated with this method when used for metals in the infrared to near ultraviolet energy region.

Liquid $\mathrm{Hg}$, cleaned by the overflow method previously used by Hodgson [23], was brought into contact with a strain-free quartz prism. Measurements were made on this system at atmospheric pressure. At all times during the measurements the surfaces could be inspected and were found free of visible contamination. The experimental method of obtaining $R_{p}, R_{s}$, and $\triangle$ was almost identical to that used by Crozier and Murphy [40]. The azimuth setting of the polarizer was fixed at $45^{\circ}$ to the plane of incidence. Two sets of intensities $I\left(0^{\circ}\right)$ and $I\left(90^{\circ}\right)$, and $I\left(45^{\circ}\right)$ and $I\left(135^{\circ}\right)$ were measured for reflection from the quartz-liquid $\mathrm{Hg}$ interface, where $I\left(A^{\circ}\right)$ is the intensity measured with the analyzer setting at $A^{\circ}$ to the plane of incidence. The liquid $\mathrm{Hg}$ was then removed and the values of the intensities $I_{0}\left(0^{\circ}\right)$ and $I_{0}\left(90^{\circ}\right)$ recorded for total internal reflection at the quartz-air interface. The angle of incidence was determined to be $60.52^{\circ}$ from the value of $\Delta$ obtained from these total reflection measurements. $R_{p}$ and $R_{s}$ were given by $R_{p}=I\left(0^{\circ}\right) / I_{0}\left(0^{\circ}\right)$ and $R_{S}=I\left(90^{\circ}\right) / I_{0}\left(90^{\circ}\right)$, 
respective]y, and $\Delta$ was determined from $[40] \cos \Delta=-\cos 2 \xi / \sin 2 \psi$, where $\tan \xi=\left[\mathrm{I}\left(45^{\circ}\right) / \mathrm{I}\left(135^{\circ}\right)\right]^{\frac{1}{2}}$ and $\tan \psi=\left[\mathrm{I}\left(0^{\circ}\right) / \mathrm{I}\left(90^{\circ}\right)\right]^{\frac{1}{2}}$. The parameter $\psi$ was actually obtained from $\tan \psi=\left[R_{p} / R_{s}\right]^{\frac{1}{2}}$.

The dielectric functions $\varepsilon_{1}$ and $\varepsilon_{2}$ were calculated from the measured ellipsometric parameters $\psi$ and $\Delta$. The values obtained for $\varepsilon_{2}$, in the form of the optical conductivity $\sigma$, are shown in Fig. 10 and those for $\varepsilon_{1}$ in Fig. 11. Also shown are previous values appearing in the literature [23, $25,27,40,43-47]$, all of which have apparently been obtained from $\psi$ and $\Delta$ under the same assumption of no surface effects. The notation $n_{0}=1.00$ indicates the data were obtained at a vacuum (or inert gas)-liquid $\mathrm{Hg}$ interface, $n_{0}=1.42$ at a cyclohexane $\left(n_{0}=1.4198\right)-1$ iquid $\mathrm{Hg}$ interface, and $n_{0}=n_{Q}$ at a quartz-liquid Hg interface. The measurements of Smith and Stromberg [47] at 2.27 eV also included $n_{0}=1.5084$ (benzene) and $n_{0}=1.3344$ (water). No differences were found in their values of $\varepsilon_{1}$ and $\varepsilon_{2}$ within their experimental accuracy of four significant figures. The Drude variations of $\sigma$ and $\varepsilon_{1}$ were calculated from

$$
\tilde{\varepsilon}_{D}=1-\frac{4 \pi \hbar \hbar \sigma_{0}}{(\tau / \hbar) E^{2}+i E}
$$

where $\tau$, the relaxation time for free carriers, was determined [44] from $\tau=\left(\mathrm{m} / \mathrm{Ne}^{2}\right) \sigma_{0}$ using the d.c. conductivity $\sigma_{0}=9.387 \times 10^{15} \mathrm{sec}^{-1}$ and the density of free carriers $\mathrm{N}=8.158 \times 10^{22} \mathrm{~cm}^{-3}$ for 1 iquid $\mathrm{Hg}$ at $20^{\circ} \mathrm{C}$. An imperfect glass-1iquid $\mathrm{Hg}$ contact $[43,44]$ apparently caused the considerable difference between the results of Lelyuk et al. [24] and those shown in Figs. 10 
and 1i. The experimental data, obtained in the various studies represented, are consistent with each other, with no systematic correlations of o or $E_{1}$ with the value of $n_{0^{*}}$. The dashed curves indicate the probable upper and lower limits of the observed quantities. It is seen that the optical properties of 1 iquid $\mathrm{Hg}$, as observed by ellipsometric measurements, are not represented by the Drude nearly-free-electron model.

The lack of any demonstrated difierences in the experimental values of $\sigma$ and $\varepsilon_{1}$ for different values of $n_{0}$ is strong evidence for the absence of any surface structure in liquid $\mathrm{Hg}$. If the surface models $[39,47]$ proposed for liquid $\mathrm{Hg}$ were applicable, then measurements made with different materials (i.e., different $n_{0}$ values) in contact with the liquid $\mathrm{Hg}$, and aralyzed assuming no surface structure, would give apparent optical properties for liquid $\mathrm{Hg}$ which varied as a function of the value of $n_{0}$.

Consider 1 iquid $\mathrm{Hg}[47]$, with $\varepsilon=-18.46+i 13.50$, covered with a $10 \AA$ thick transparent layer of refractive index $n_{1}=1.45$ as shown in Fig. 12 . As defined by Burge and Bennett [48], we call the dielectric function $\varepsilon$, calculated from the measured ellipsometric parameters $\psi$ and $\Delta$ for a real surface under the assumptions that the surface is smooth, homogeneous, and isotropic, the pseudodielectric function. If surface structure is present on the real surface, then the pseudodielectric function will, in general, be a function of $\phi$ and $n_{0}$, the angle of incidence and the refractive index of the ambient medium, respectively. The calculated pseudodielectric functions for the chosen example at a photon energy of $2.27 \mathrm{eV}$, are shown as functions of $\phi$ and $n_{0}$ in Fig. 12. For $\phi=70^{\circ}$, the variations of $\varepsilon_{1}$ and $\varepsilon_{2}$ with $n_{0}$ are large enough to indicate experimentally the existence of the surface structive. 
Most ellipsonetric data are taken at an angle of incidence in the region of $70^{\circ}$ $\left( \pm 10^{\circ}\right)$ and thus, if there is a surface layer on liquid $\mathrm{Hg}$ with properties resembling those shown in Fig. 12, it should show up as systematic differences in $\sigma$ and $\varepsilon_{1}$ with $n_{0}$ in Figs. 10 and 11 . Other reasonable values of $n_{1}$ and thickness of the surface layer lead to the same conclusion. Thus lack of systematic variations in $\sigma$ and $\varepsilon_{1}$ with the value of $n_{0}$ implies that there is no surface layer or surface inhomogeneity on liquid $\mathrm{Hg}$. A similar analysis [42] rules out any anisotropy in the optical properties of liquid $\mathrm{Hg}$ in the vicinity of its surface.

The pseudodielectric function $\varepsilon\left(n_{0}, \phi\right)$ can be used in several other tests for surface structure. For example, a value of $\varepsilon\left(n_{0}, \phi\right)$ obtained for a real surface at a given photon energy can be used to calculate the anticipated variation of the reflectance at that energy as a function of the refractive index of the ambient medium, $n_{0}{ }^{\prime}$, and the angle of incidence $\phi^{\prime}$. This quantity, $R_{a n t}\left(n_{0}{ }^{-}, \phi^{-}\right)$can then be compared with the experimentally observed quantity, $R_{\exp }\left(n_{0}{ }^{-}, \phi^{\prime}\right)$. Assuming the system shown in Fig. 12 with $n_{0}=1$ and $\phi=70^{\circ}$, the quantities $R_{\text {ant }}\left(n_{0}^{-}, \phi^{-}\right)$and $R_{\exp }$ $\left(n_{0}{ }^{\infty}, \phi^{-}\right)$have been calculated. The difference $\delta R=R_{a n t}\left(n_{\sigma^{-}}, \phi^{\prime}\right)-R_{\exp }$ $\left(n_{0}{ }^{\prime}, \phi^{\prime}\right)$ has been plotted in Fig. 13 which shows $\delta R_{p}$ and $\delta R_{s}$ as functions of $n_{0}^{\prime}$ and $\phi^{\prime} . \delta \psi^{\prime}$ and $\delta \Delta$, calculated for the same system, are also shown in Fig. 13. It is seen that $\delta R_{p}$ or $\delta \Delta$, for $n_{0}{ }^{\prime} \neq n_{0}$ and large values of $\phi^{\prime}$ are large enough to be detected, if surface structure exists.

The present measurements of $R_{p}$ on liquid ilg were used to perform a $\delta R_{p}$-check. For this purpose the availabie pseudodielectric functions obtained with $n_{0}=1$ at each energy (Figs. 10 and 11) were used to calculate the anticipated 
value of $R_{p}$ for $n_{0}{ }^{\prime}=n_{Q}$ and $\phi^{\prime}=60.52^{\circ}$, i.e., the actual experimental con ditions of our measurement of $R_{p}$. The anticipated and measured values of $R_{p}$ for our present studies on liquid $\mathrm{Hg}$ are shown in the top part of Fig. 14 . Also shown in the lower part of Fig. 14 is a similar comparison for the measurements of Crozier and Murphy [40]. In both studies, values of $R_{p}$ obtained by direct measurement at the quartz-liquid $\mathrm{Hg}$ interface and the corresponding calculated quantity both show a systematic deviation from the Drude values, but are in good agreement with each other.

Further tests for the detection of surface structure from ellipsometric measurements involve searching for inconsistencies in the values of $R_{p}, R_{s}$, and $\Delta$ measured simultaneously at a given interface. One method involves comparing one of these measured quantities with its value as calculated from the other two. Another method involves calculating the optical constants from three different pairs of the measured quantities. These tests have been described previously [42]; none show any evidence for surface structures in liquid lig.

The apparent Drude optical properties of liquid $\mathrm{Hg}$, which have been extracted from two or more reflectance measurements at a given energy, and which have been thought to be in conflict with the ellipsometric measurements, can be shown [42] to be unreliable because of the large uncertainties involved in the reflectance measurements relative to the accuracy necessary to obtain reliable optical properties in this energy range. Figure 15 shows plots in the $\varepsilon_{7}-\varepsilon_{2}$ plane of calculated equireflectance curves. For each curve the type of reflectance measurement is specified by a subscript $(\bar{R}=$ reflectance at $\phi=45^{\circ}$ for unpolarized light), the value of the ambient 
refractive index is given, and the stated value of the reflectance has been obtained from the Drude values of $\varepsilon_{1}$ and $\varepsilon_{2}$ for liquid $\mathrm{Hg}$ at $2.27 \mathrm{eV}$. Also indicated on each curve is an error bar of length $2 R_{\text {err }}$ which corresponds to a typical range of the experimental uncertainty reported in the literature. This error bar specifies a path $2 R_{\text {err }}$ wide centered on the equireflectance curve. When two experimental values of reflectance, such as $R_{N}\left(n_{0}=1.0\right)$ and $R_{N}\left(n_{0}=1.4\right)$, at $2.27 \mathrm{eV}$ are used to determine $\varepsilon_{1}$ and $\varepsilon_{2}$ for liquid $\mathrm{Hg}$, the values obtained from the intersection of the equireflectance curves will lie within the overlap region of the corresponding paths. Because the equireflectance curves are almost paralle1, the region of overlap for any two of the types of reflectance measurements shown in Fig. 15 extends from the top of the graph to beyond the bottom. This demonstrates that optical properties obtained from reflectance measurements have large associated errors: in the case of Fig. 15, depending on the pair of reflectances, this error may be $\sim 60$ to $2700 \%$. At lower photon energies the uncertainties associated with optical properties determined by this method are even larger. Bloch and Rice [39] report $R_{N}$ for $v$ "values of $n_{0}$ while Mueller [49] reports $R_{N}$ and $R_{S}$ at $\phi=45^{\circ}$ for lrquid $\mathrm{Hg}$ in contact with sapphire. In both cases, they contend that since they observe values of reflectance consistent with the Drude values of $\varepsilon_{1}$ and $\varepsilon_{2}$ that this proves the validity of the Drude values. We have shown that there is a very large uncertainty in the optical properties when they are determined from reflectance measurements, and that observing "Drude" reflectances does not necessarily imply Drude values of the optical properties. In fact, we demonstrate in 
Fig. 15 that the reflectances measured by Bloch and Rice and by Muller are not inconsistent with the values of $\varepsilon_{1}$ and $\varepsilon_{2}$ obtained by ellipsometry for 1 iquid $\mathrm{Hg}$ at $2.27 \mathrm{eV}$. These values, using the same notation as in Fig. 10, are shown in Fig. 15 together with a rectangle of dashed-lines indicating the probable upper and lower limits. In order to determine the optical constants by reflectance measurements with accuracy comparable to that of the ellipsometric results, reflectances would have to be measured ten to a hundred times more accurately than in the experiments so far reported in the literature. In fact, the high correlation between the determination of the optical properties from reflectance measurements and the consistency of those properties with the Drude theory seems to stem from the a priori assumption, in some cases, of the validity of Drude theory.

of the reported reflectance measurements, only those of Schulz [29] do not fall in the above category. Schulz obtained values of the optical properties from measurements of $\bar{R}$ and $\theta$, the phase change for reflectance at normal incidence. In a $\varepsilon_{1}-\varepsilon_{2}$ plane, equi- $\vec{R}$ and equi- $\theta$ curves cross nearly at right angles and experimental errors introduce only smal7 errors into the values determined for $\varepsilon_{1}$ and $\varepsilon_{2}$. Similar considerations apply to equi- $\psi$ and equi- $\Delta$ curves which are employed in the ellipsometric determination of the optical properties. Schulz's experiments appear to have been performed to a high standard of experimental accuracy and examination of his published papers does not reveal any apparent reason to doubt the "Drude" values which he obtained [42]. However, detailed KramersKronig analyses, over a wide energy range [42], show that Drude optical properties are not possible for liquid $\mathrm{Hg}$, whereas the optical properties 
obtained from ellipsometric measurements are internally consistent with the available data on liquid Hg above the limit of the ellipsometric measurements at $\sim 5 \mathrm{eV}$.

The Kramers-Kronig relation used was that for the phase change on refiection at normal incidence

$$
\theta(E)=-\frac{1}{\pi} \int_{0}^{\infty} \frac{E \ln R_{N}\left(E^{\prime}\right)}{\left(E^{\prime}\right)^{2}-E^{2}} \mathrm{dE}^{\prime} .
$$

If $R_{N}\left(E^{-}\right)$and $\theta(E)$ are calculated from the pseudodielectric functions obtained from ellipsometric measurements on real surfaces on the assumption that there is no surface structure, this Kramers-Kronig relation would appear to break down in the presence of surface structure. However, this test for the presence of surface structure cannot be applied as experimental values of the pseudodielectric function are not available over the whole energy spectrum. We can use this relation to demonstrate that the Drude values of $\theta$ obtained by Schulz are highly unlikely, whereas the optical properties determined from ellipsometric measurements are probably correct.

The measured values of the normal incidence reflectance, $R_{N}$, obtairied by Wilson and Rice [30] from 2 to $20 \mathrm{eV}$ are shown in Fig. 16. Wilson and Rice analyzed these data through the Kramers-Kronig relation by assuming the Drude form for $R_{N}$ below $2 \mathrm{eV}$ and an analytic extrapolation of the measured $R_{N}$ to energies above $20 \mathrm{eV}$. They obtained values of the optical properties from 2 to $20 \mathrm{eV}$ which are quite close to the Drude values in the region below $4 \mathrm{eV}$. This analysis was somewhat circuitous, however, since 
the extrapolation of $R_{N}$ to high energies was chosen to give the Drude value of $\theta$ at $2.0 \mathrm{eV}$. In an attempt to obtain a more realistic extrapolation of $R_{N}$ above $20 \mathrm{eV}$, in the absence of optical data for either liquid or solid $\mathrm{Hg}$, we have used the optical data available in the literature [50] up to $900 \mathrm{eV}$ for two materials with atomic numbers close to that of $\mathrm{Hg}$ $(Z=80)$ to estimate values of $R_{N}$ for $\mathrm{Hg}$. From solid $\mathrm{Au}(Z=79)$,

$$
R_{N, H g}=\left(D_{H g} / D_{A u}\right)^{2} R_{N, A u}=0.476 R_{N, A u}
$$

where $D_{\mathrm{Hg}}$ and $D_{\mathrm{Au}}$ are the atomic densities in liquid $\mathrm{Hg}$ and solid $\mathrm{Au}$, respectively. Sinilarly, from solid $B i(Z=83)$,

$$
R_{N, H g}=\left(D_{H g} / D_{B i}\right)^{2} R_{N, B i}=2.077 R_{N, B i}
$$

These estimated values of $R_{N}$ are shown in Fig. 16 and appear to agree reasonably well with each other above $\sim 50 \mathrm{eV}$. This agreement demonstrates that this is a reasonable method of determining $R_{N}$ for high $z$ materials at high enough energies, hence removing a major part of the uncertainty involved in the extrapolations used by Wilson and Rice. Above $900 \mathrm{eV}$ we extrapolated $R_{N}$ according to an $E^{-4}$ dependence.

The results of our Kramers-Kronig analyses are demonstrated in Fig. 17 in the form of the difference between $\theta(E) / E$. calculated from the $R_{N}$ values shown in Fig. 16 and the corresponding Drude values. The two solid lines were obtained, in this way, from the $R_{N}$ values of Wilson and Rice [30] from 2 to $20 \mathrm{eV}$ in combination with the $R_{\mathrm{N}}$ values of solid $\mathrm{Au}$ and solid $B i$, respectively. The two dashed lines were obtained from $R_{N}$ calculated from optical properties obtained from ellipsometric measurements 
from 0.2 to $4 \mathrm{eV}$ in combination with the $R_{f}$ values of $W i l$ son and Rice from 5 to $20 \mathrm{eV}$ and the $R_{N}$ values of $A u$ and $B i$, respectively. Also shown in Fig. 17 are $\theta(E) / E$ - $\theta_{D}(E) / E$ calculated direct?y from the optical properties of liquid $\mathrm{Hg}$ obtained from ellipsometric measurenents as summarized in Figs. 10 and 11 and from the "Drude values" of $\theta(E)$ obtained by Schu]z [29]. It is seen that the calculated values of $\theta(E) / E-\theta_{0}(E) / E$ obtained from ellipsometric measurements lie essentially within the dashed curves. Thus, the variations with energy of the piotted function of $\theta$ obtained through a Kramers-Kronig analysis are consistent with the ellipsometric optical properties of liquid $\mathrm{Hg}$. Just as the estimated $R_{N}$ values using the $A u$ and $B i$ data between 20 and 50 ev obviously set. upper and lower linits, respectively, on the $R_{N}$ for liquid Ha in this energy range, the dashed curves in Fig. 17 obtained from ellipsometric $R_{N}$, set reasonable 7 imits on the ellipsometric data. By a proper choice of $R_{N}$ between 20 and $50 \mathrm{eV}$ it is obviously possible to reproduce the ellipsometric values of $\theta$ from the ellipsometric values of $R_{N}$ using the Kramers-kronig relation for $\theta(E)$.

On the other hand, the "Drude values" of Schulz are inconsistent with a Kramers-Kronig analysis employing any reasonable extrapolation. One possible explanation for this discrepancy is that the Ag film which Schulz deposited on a mica film in order to make a good mica-Hg contact did not dissolve away completely as expected.

In conclusion, it has been shown that the optical properties obtained from ellipsometric measurements are representative of bulk liquid $\mathrm{Hg}$. No evidence is seen in any of the experimental data on liquid $\mathrm{Hg}$.. une 
existence of surface structure or anomalous sumface properties. The optical properties of liquid Hg are not Drude-lite. The only known attempt to explain deviations from the simple Drade theory in liquid $\mathrm{Hg}$ is due to Smith [51]. Previously, with und:1e Eiphas given to reflectance measurements and the expectation of Drude belario:", the deviations shown by ellipsometric neasurements wert suspect. Since it has now been established that the ellipsometric valies are probably correct, there is a need for ne: theoretical efiorts to explain the nonDrude behavior of liquid Hg. Acknouledgment

Research sponsored by the Office of lieal th ard Enviromental Research, U. S. Departrient of Energy, under contrac $4-745-e n g-20$ with the union Carbide Corporation. 


\section{References}

1. D. E. Aspnes, in: Optical Properties of Solids; liew Developments, Ed. B. O. Seraphin (Horth-Holland, Aristerdan, 1976) p. 799.

2. R. M. A. Azzan and N. M. Bashara, Ellipsometry and Polarized Light (North-Holland, Ansterdam, 1977).

3. Ellipsometry in the Measurement of Surfaces and Thin Filisis, Eds. E. Passaglia, R. R. Strombery and J. Kruger, ilBS Misc. Publ. 256 (U.S. GPO, Washington, D.C., 1964).

4. Recent Developments in Ellipsonetry, Eds. M. H. Bashara, A. B. Burkman and A. C. Hall (North-Holland, Ansterdan, 1969).

5. Third International Conference on Ellipsonetry, Fds. M. M. Bashara and P. M. A. Azzam (fiorth-Holland, Arnsterdan, 1976).

6. A. Rothen, in: Progress in Surface and Hembrane Science, Vol. 8, Eds. D. A. Cadenhead, 1. F. Danielli and 11. D. Posenherg (Academic Press, New York, 1974) p. 81.

7. G. T. Ryoub and N. M. Bashara, 3. Opt. Soc. Am. 68 (1978) 978.

8. H. F. Hazebroek and A. A. Holscher, Proc. 6th Intern1. Vacuum Congr. 1974. Japan J. App1. Phys. Suppl. 2, pt. 1 (1974) 673.

9. F. Abelès, Surf. Sci. 56 (1976) 237.

10. E. C. Chan and J. P. Marton, J. Appl. Phys. 43 (1972) 4027.

11. K. Kinosita and M. Yamamoto, Thin Solid Films 34 (1976) 283.

12. N. V. Smith, Phys. Rev. Lett. 21 (1968) 96; Phys. Rev. 183 (1969) 634.

13. H. Mayer and B. Hietel, Optical Properties and Electronic Structure of Metals and Alloys, Ed. F. Abeles (North-Holland, Amsterdam, 1966) p. 47 . 
14. T. Inagaki, L. C. Emerson, E. T. Arakawa and M. H. Williams, Phys. R.ev. B13 (1976) 2305.

15. G. K. T. Conn and G. K. Eaton, J. Opt. Soc. Am. 44 (1954) 546.

16. S. N. Jasperson and S. E. Sclmatterly, Rev. Sci. Instrun, 40 (1969) 761.

17. A. G. Mathewson and H. P. Myers, Philos. Mag. 25 (1972) 853.

18. 0. Hunderi, Surf. Sci. 57 (1976) 451.

19. H. P. Myers and P. Sixtensson, J. Phys. F: Metal Phys. 6 (1976) 2023.

20. M. Rasigni and G. Rasigni, J. Opt. Soc. Am. 67 (1977) 54.

21. T. A. Callcott and E. T. Arakawa, J. Opt. Soc. Am. 64 (1974) 839.

22. C. V. Kent, Phys. Rev. 14 (1919) 459.

23. J. H. Hodgson, Phil. Mag. 4 (1959) 183; 5 (1960) 272;6 (1961) 509; 7 (1962) 229;8 (1963) 735.

24. L. G. Lelyuk, I. N. Shklyarevskii and R. G. Yarovaya, Opt. Spectrosc. $16(1964) 263$.

25. iv. V. Smith, Adv. Phys. 16 (1967) 629.

26. J. C. Miller, Phil. Mag. 20 (1969) 1115.

27. iN. R. Comins, Phii. Mag. 25 (1972) 817.

28. T. Inagaki, E. T'. Arakawa, R. D. Birkoff and M. W. Williams, Phys. Rev. B13 (1976) 5610.

29. L. G. Schulz, Adv. Phys. 6 (1957) 102.

30. E. G. Wilson and S. A. Rice, Phys. Rev. 145 (1966) 55.

31. N. H. March, Can. J. Chem. 55 (1977) 2165.

32. N. E. Cusack, in: The Properties of Liquid Metals, Ed. S. Takeuchi (John Wiley, New York, 1973) p. 157.

33. W. D. Knight and A. G. Berger, Ann. Phys. 8 (1959) 173.

34. N. V. Smith, Phys. Rev. 163 (1967) 552. 
35. J. S. Helman and H. Baltensperger, Phys. Rev. B15 (1977) 4109.

36. J. P. Petrakian, A. R. Cathers, J. E. Parks, R. A. IfacRae, T. A. Callcott and E. T. Arakawa, Submitted to Phys. Rev.

37. R. A. MacRae, E. T. Arakawa and M. H. Williams, Phys. Rev. 162 (1967) $6\rceil 5$.

38. T. E. Faber, Introduction to the Theory of Liquid Metals (Cambridge University Press, Cambridge, 1972).

39. A. N. Bloch and S. A. Rice, Phys. Rev. 185 (1969) 933.

40. E. D. Crozier and E. Murphy, Can. J. Phys. 50 (1972) 1914.

41. D. Guidotti and S. A. Rice, Phys. Rev. B15 (1977) 3796.

42. T. Inagaki, E. T. Arakawa and M. W. Williams, Submitted to Phys. Rev.

43. T. Smith, J. Opt. Soc. Aln. 57 (1957) 1207.

44. T. E. Faber and N. V. Smith, J. Opt. Soc. Am. 58 (1968) 102.

45. G. Busch and J. Guggenheim, Helv. Phys. Acta 41 (1968) 401.

46. H. J. Choyke, S. H. Yosko and T. H. O'Keeffe, Solid State Commun. 9 (1971) 361 .

47. L. E. Smith and R. R. Stromberg, J. Opt. Soc. Am. 56 (1966) 1539.

48. D. K. Burge and H. E. Bennett, J. Opt. Soc. Am. 54 (1964) 1428.

49. W. E. Mueller, J. Opt. Soc. Am. 59 (1969) 1246.

50. H. -J. Hagemann, W. Gudat and C. Kunz, Report DESY SR-74/7 (1974).

51. N. V. Smith, Phys. Lett. A26 (1968) 126. 


\section{Figure Captions}

1. Optical conductivity vs incident photon energy of solid $\mathrm{Na}$ at room temperature. References to the data shown are given in Ref. 14.

2. Optical conductivity of solid Na obtained by Inagaki et al. [14] and Smith [12].

3. Values of $\beta$ defined by $\psi=45^{\circ}-\beta$ for a quartz-Na interface. The lower open circles are Smith's data [12] obtained by the null method for a single reflection at an incidence angle of $75^{\circ}$ and the upper open circles are $4 \beta$ obtained from four reflections. The filled circles are the data of Inagaki et al. [14] obtained by the photometric metiod for a single reflection at an incicience angle of $70^{\circ}$.

4. Real part of the dielectric constant of solid Na [14].

5. Optical conductivity vs incident photon energy of solid Li at room temperature.

6. Optical constants of solid Li obtained by Rasigni and Rasigni [20], Ca17cott and Arakawa [21] and Inagaki et a1. [14].

7. Optical conductivity vs incident photon energy of liquid $\mathrm{Na}$ obtained by Inagaki et al. [28] and Mayer and Hietel [13].

8. Dielectric functions of liquid $S n$ vs incident photon energy. Drude curves for Hodgson's data and for our recent results have been calculated using parameters quoted in Ref. 36.

9. The plot of $\left(1-\varepsilon_{\eta}\right)^{-1}$ vs $\lambda^{-2}$ for liquid $\mathrm{Sn}$ at $261^{\circ} \mathrm{C}$, with $\lambda$ in $\mu \mathrm{m}$.

10. Optical conductivity vs incident photon energy of liquid $\mathrm{Hg}$ at room temperature. All values were obtained from ellipsometric measurements, as explained in the text. The dashed lines indicate the probable upper and lower limits of the observed conductivities. 
11. Values of $\varepsilon_{7}$ vs incident photon energy for liquid $\mathrm{Hg}$ obtained from ellipsometric measurements. The different symbols refer to the references indicated in Fig. 10.

12. The pseudodielectric functions $\varepsilon_{1}$ and $\varepsilon_{2}$ obtained at $2.27 \mathrm{eV}$ for liquid $\mathrm{Hg}$ covered with a $10 \AA$ thick transparent layer of refractive index 1.45 as functions of the angle of incidence $\phi$ and the refractive index, $n_{0}$, of the ambient medium. The dielectric constants assumed for $\mathrm{Hg}$ are from Smith and Stromberg [47].

13. Differences between the anticipated and experimental values of $R_{p}$, $\mathrm{R}_{s}, \psi$, and $\Delta$ as functions of $n_{0}^{*}$ and $\phi^{*}$ for the system shown in Fig. 12. The anticipated values were obtained from the pseudodielectric function $\varepsilon\left(n_{0}=1, \phi=70^{\circ}\right)$.

14. Values of $R_{p}$ measured at a quartz-liquid $H a$ interface compared with those anticipated from the values of the dielectric function measured at a free $\mathrm{Hg}$ surface.

15. Equireflectance curves $R_{N} ! \bar{R}$, and $R_{S}$ presented in the $\varepsilon_{7}-\varepsilon_{2}$ plane. The reflectance values are those corresponding to the Drude values of the optical properties of liquid $\mathrm{Hg}$ at $2.27 \mathrm{eV}$. The type of reflectance and the refractive index, $n_{0}$, of the ambient medium are indicated. The values of $\varepsilon_{7}$ and $\varepsilon_{2}$ obtained from ellipsometric measurements at $2.27 \mathrm{eV}$ are shown using the same symbols as in Fig. 10. The dashed-1ine rectangle corresponds to the probable upper and lower limits of the ellipsometric data as indicated in Figs. 10 and 11 .

16. The $R_{N}$ measured by Wilson and Rice [30] at a vacuum-liquid Hg interface combined with values of $R_{\mathrm{N}}$ calculated from the experimental optical properties of solid $\mathrm{Au}$ and $\mathrm{Bi}$ [50].

17. The phase shift on reflection $\theta(E)$ divided by the photon energy $E$ relative to the Drude values calculated from a Kramers-Kronig analysis of $R_{N}$ for the vacuum-liquid $\mathrm{Hg}$ interface and from the dielectric function $\varepsilon$. 


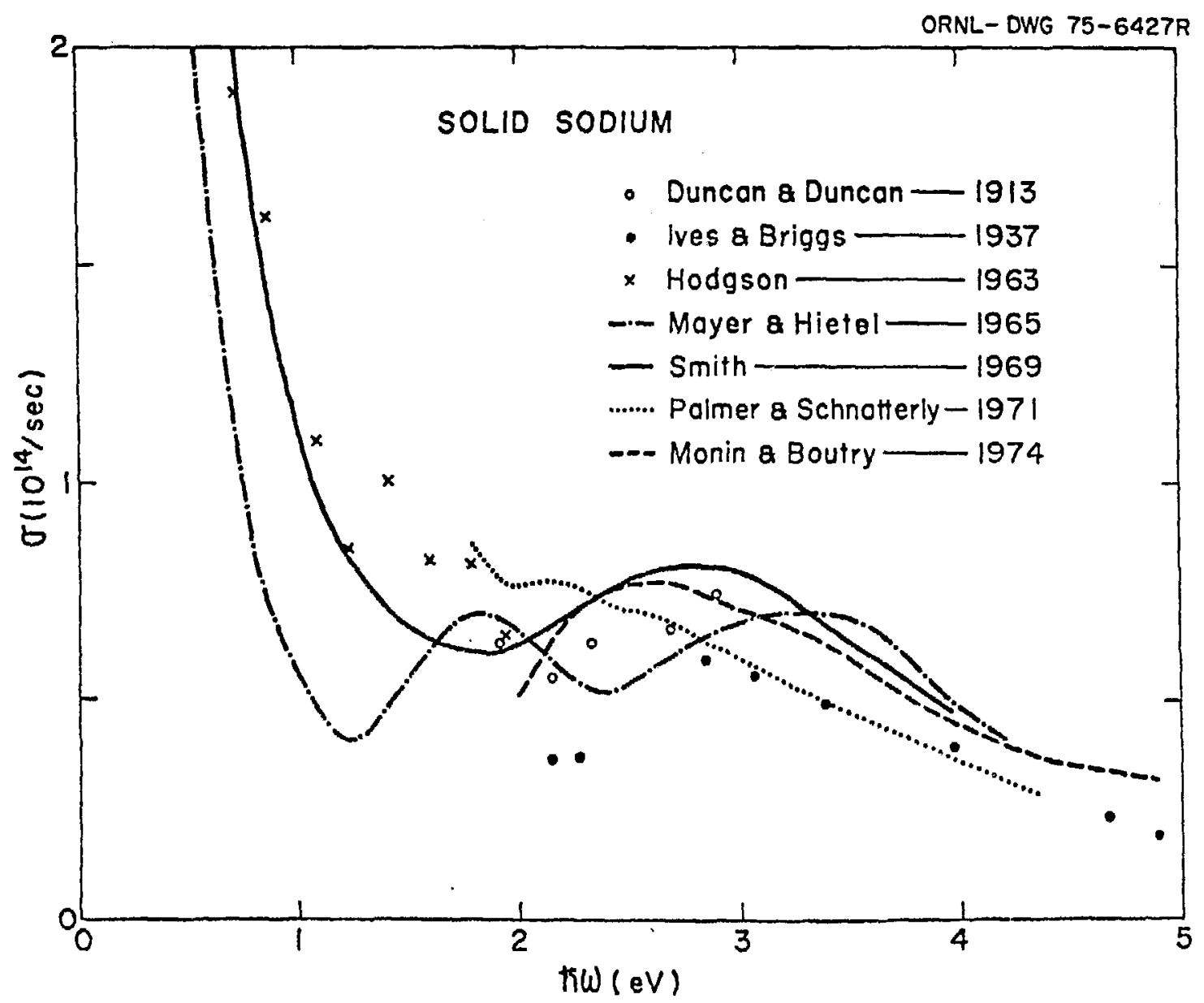




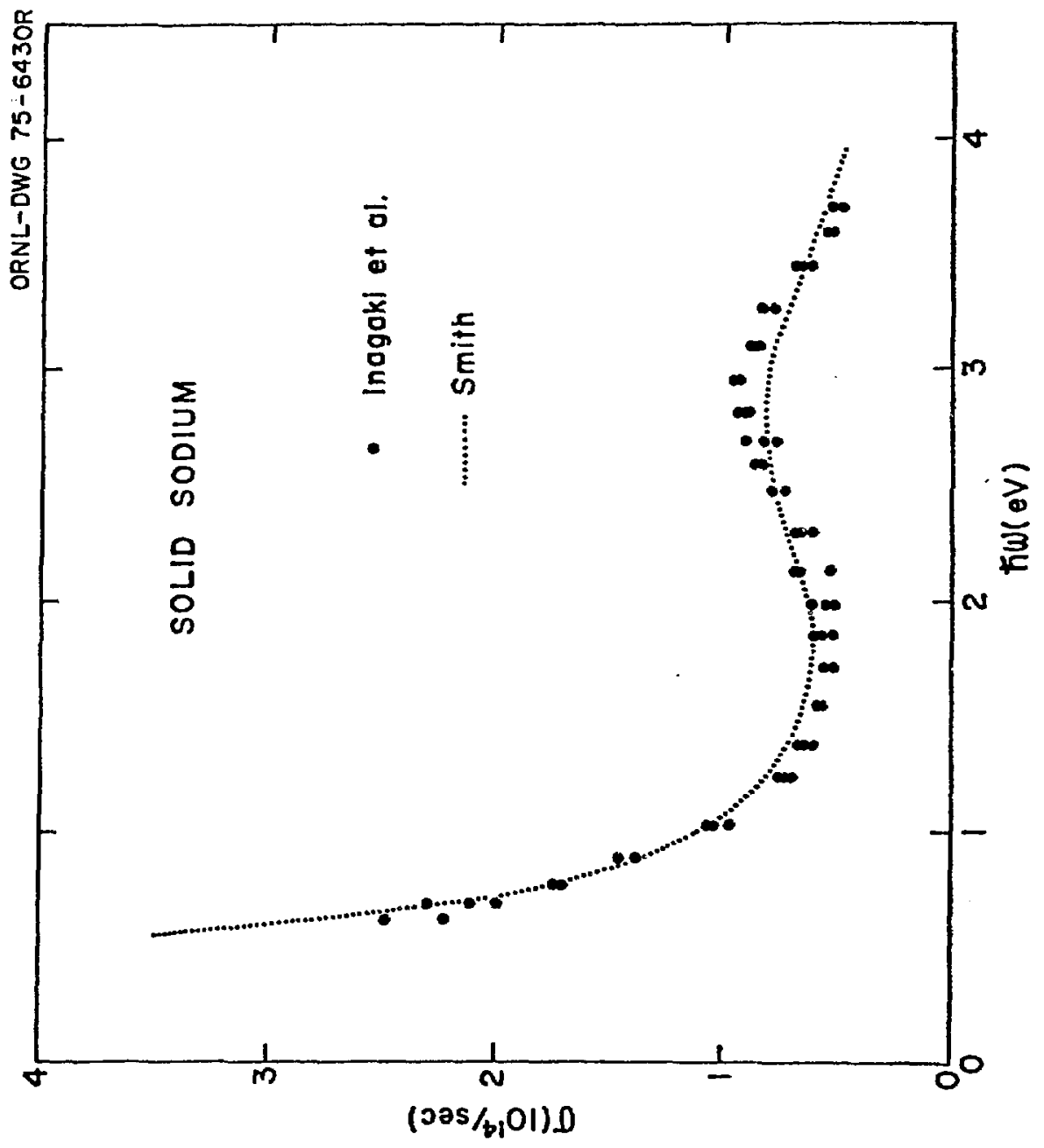

Figure 2 


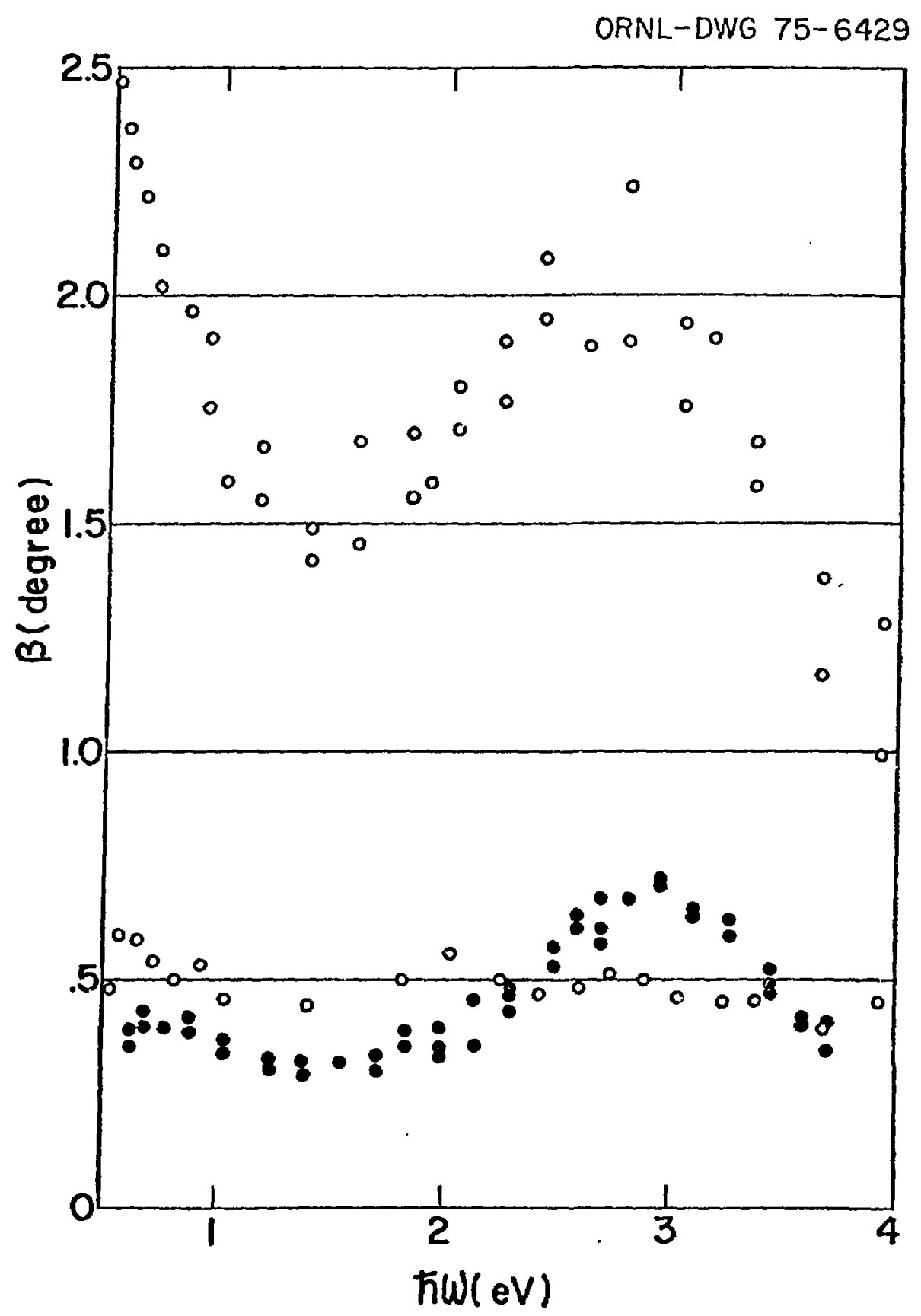

Figure 3 


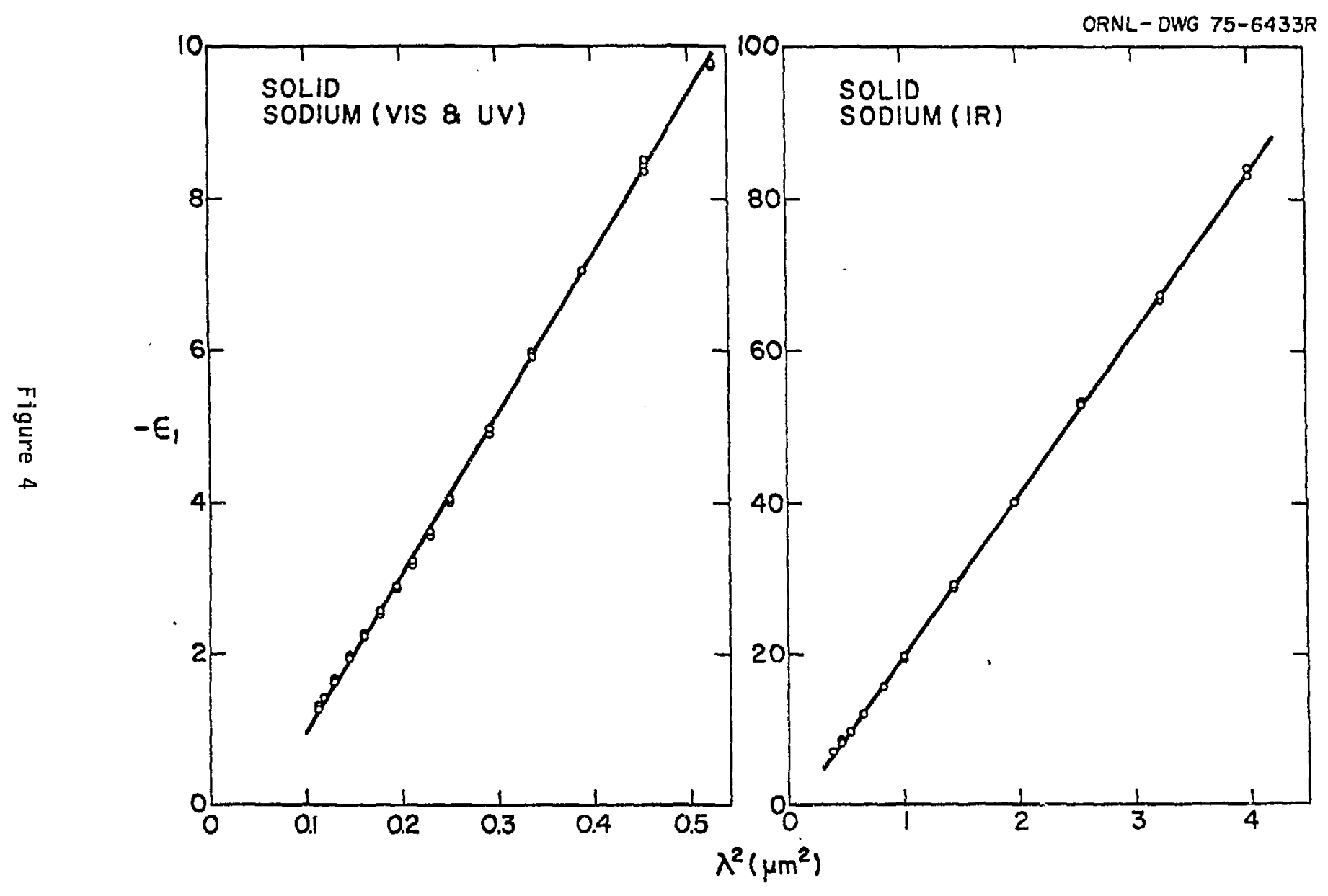




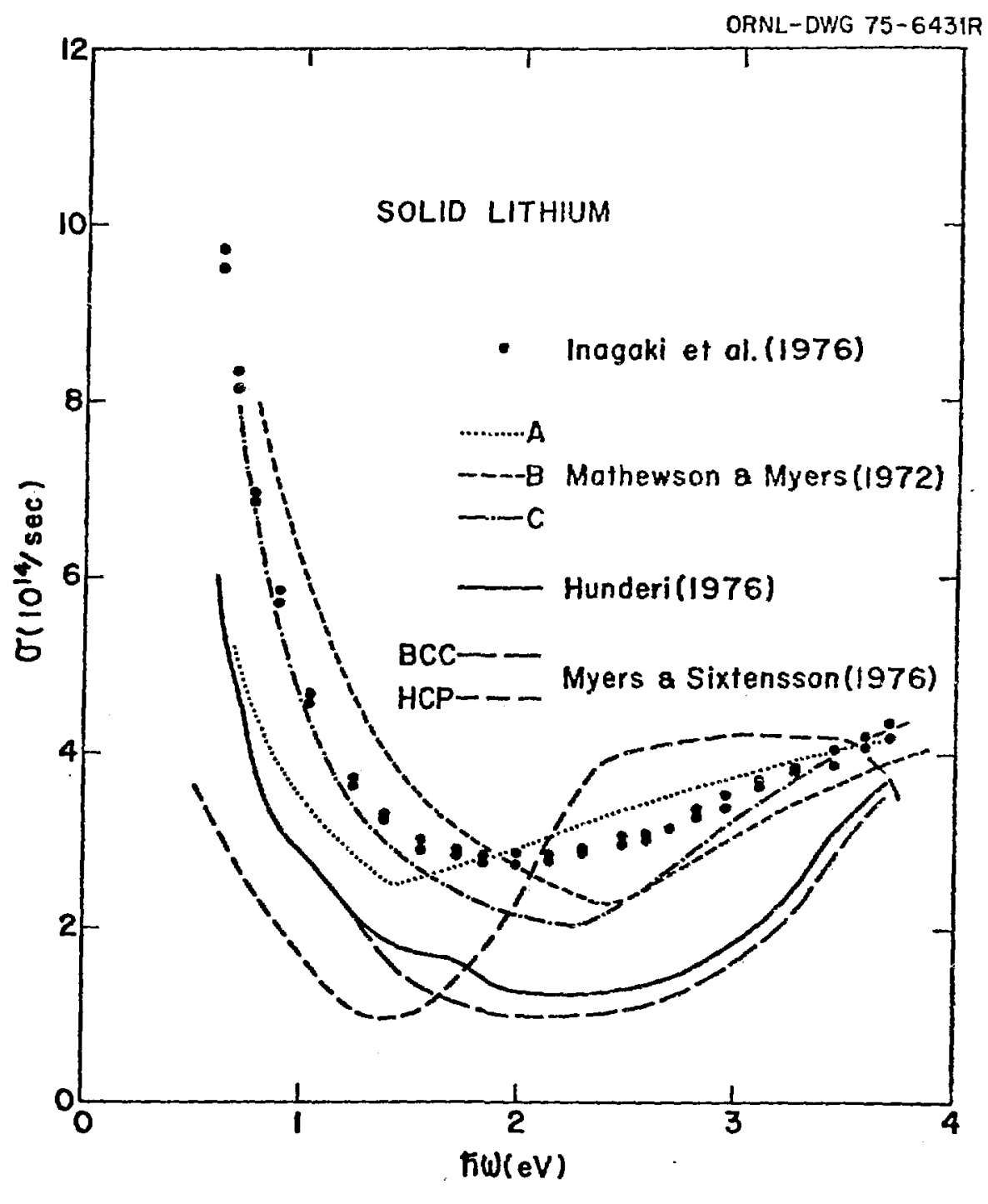

Figure 5 


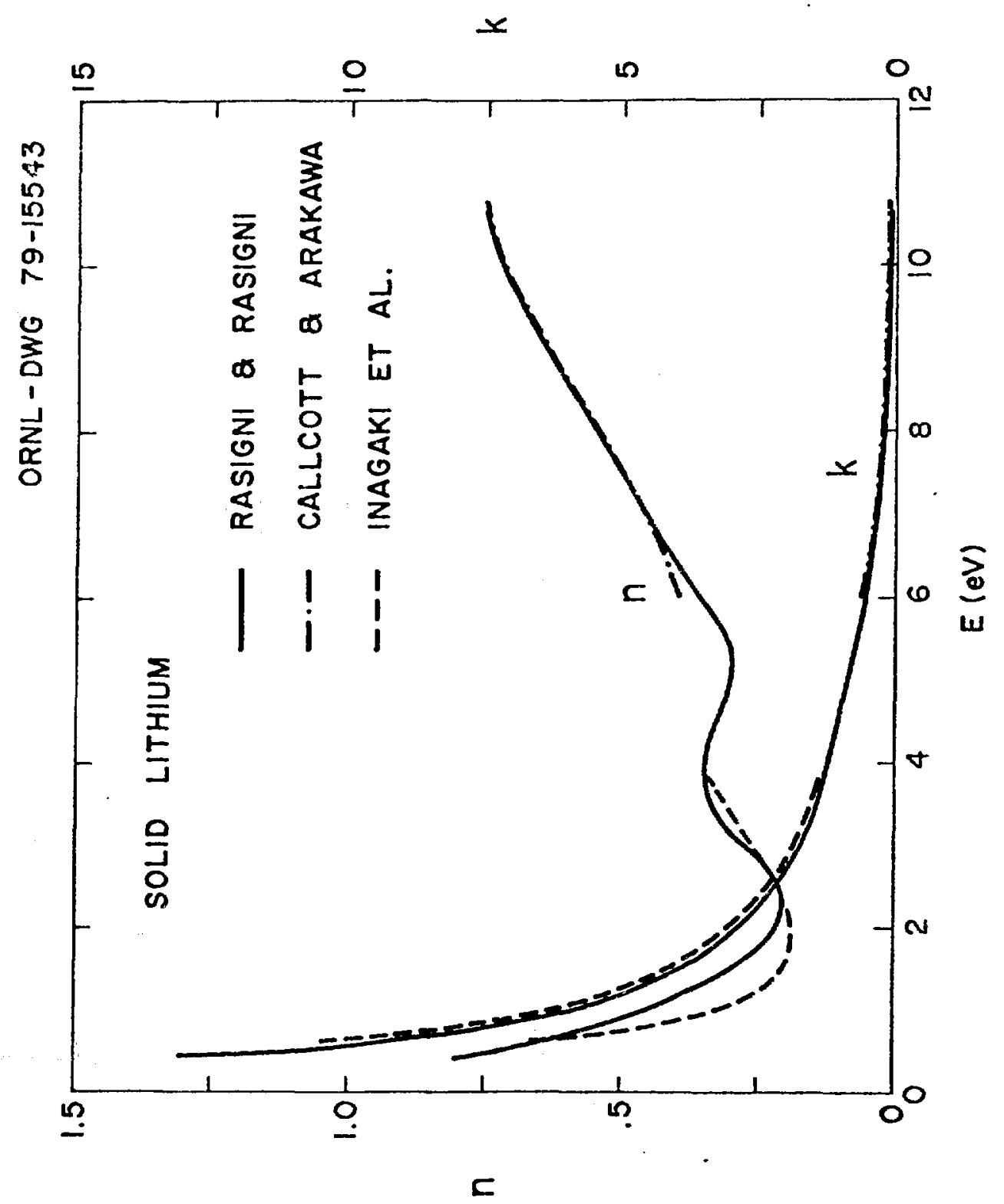

Figure 6 


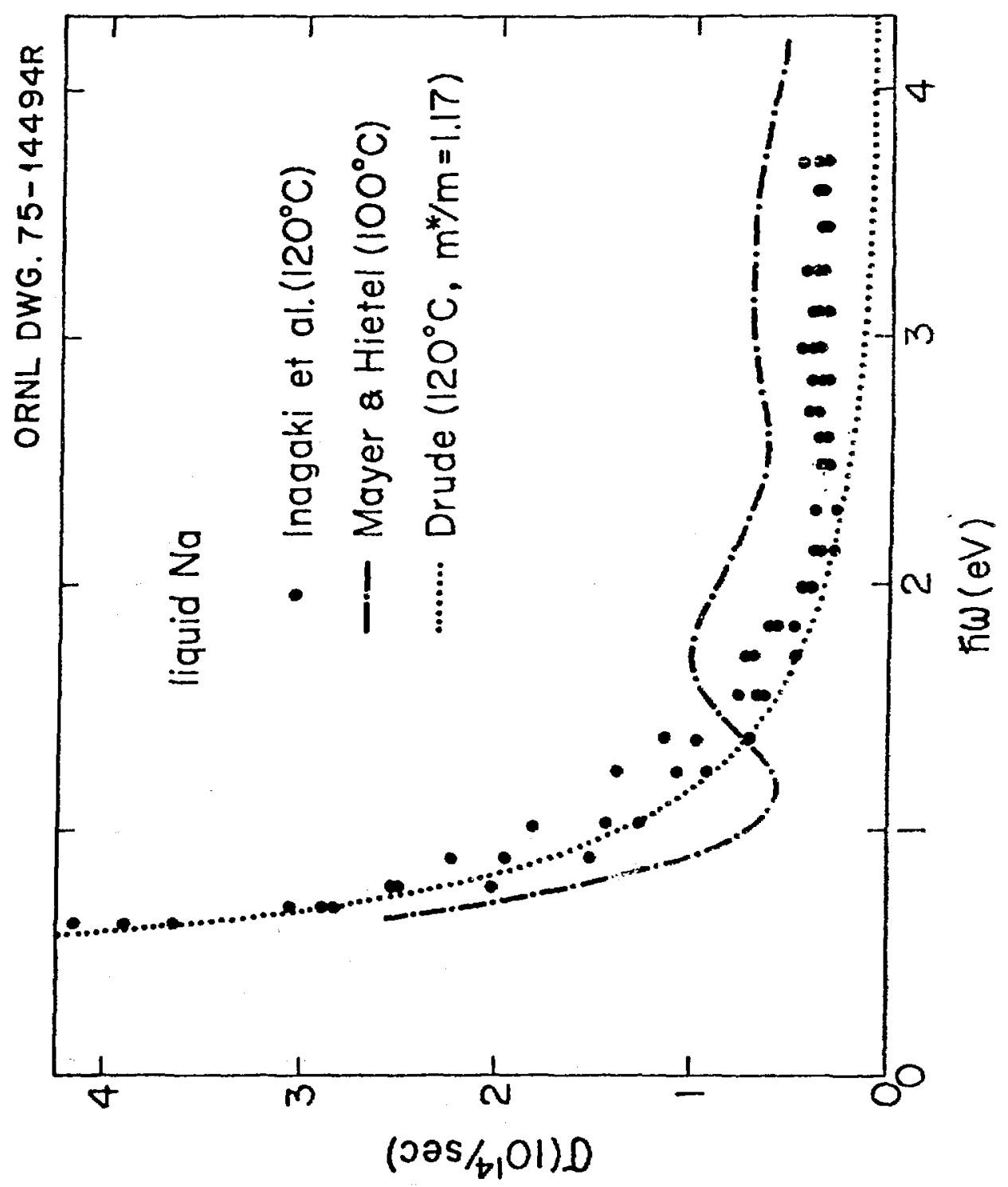

Figure 7 


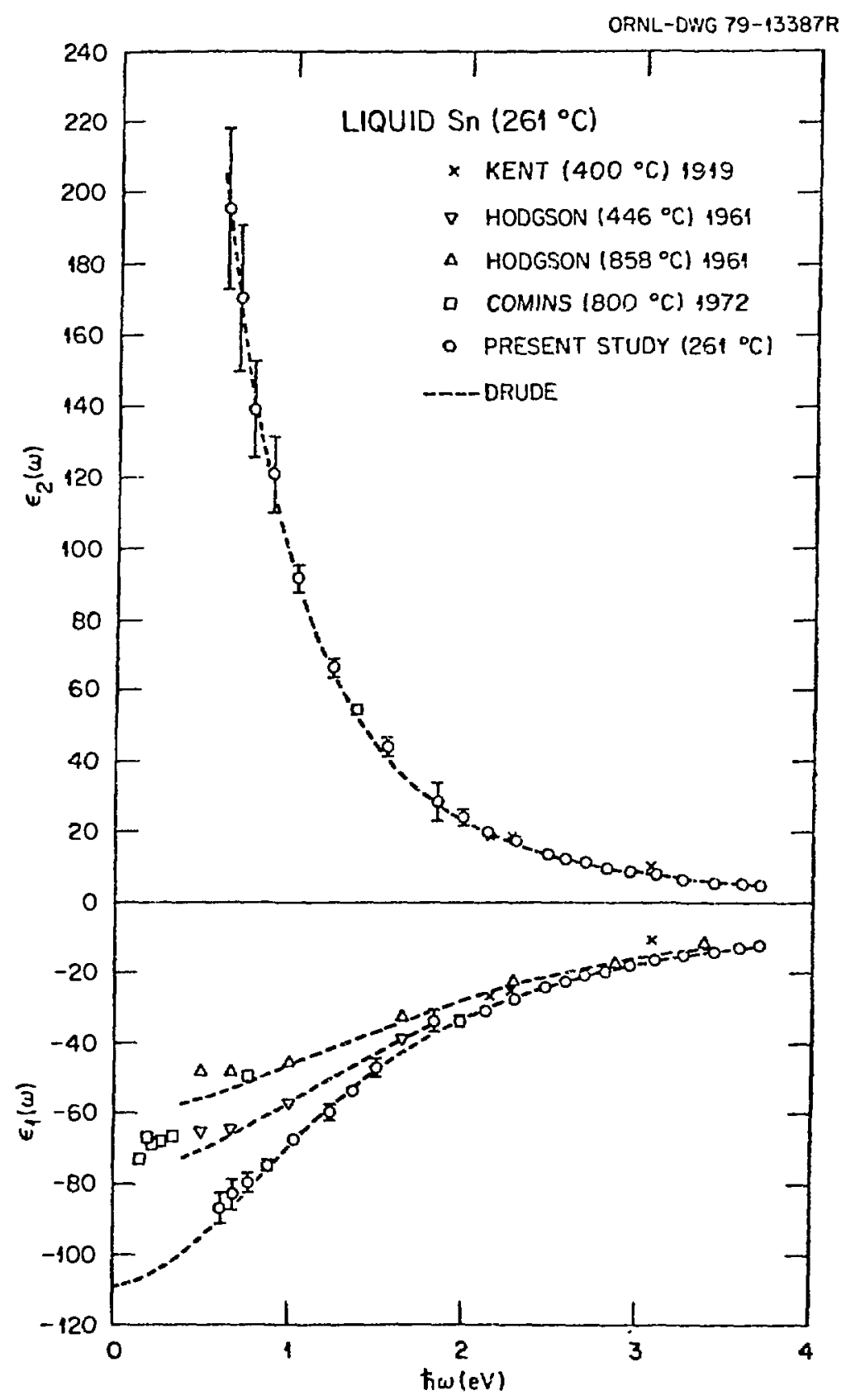

Figure 8 


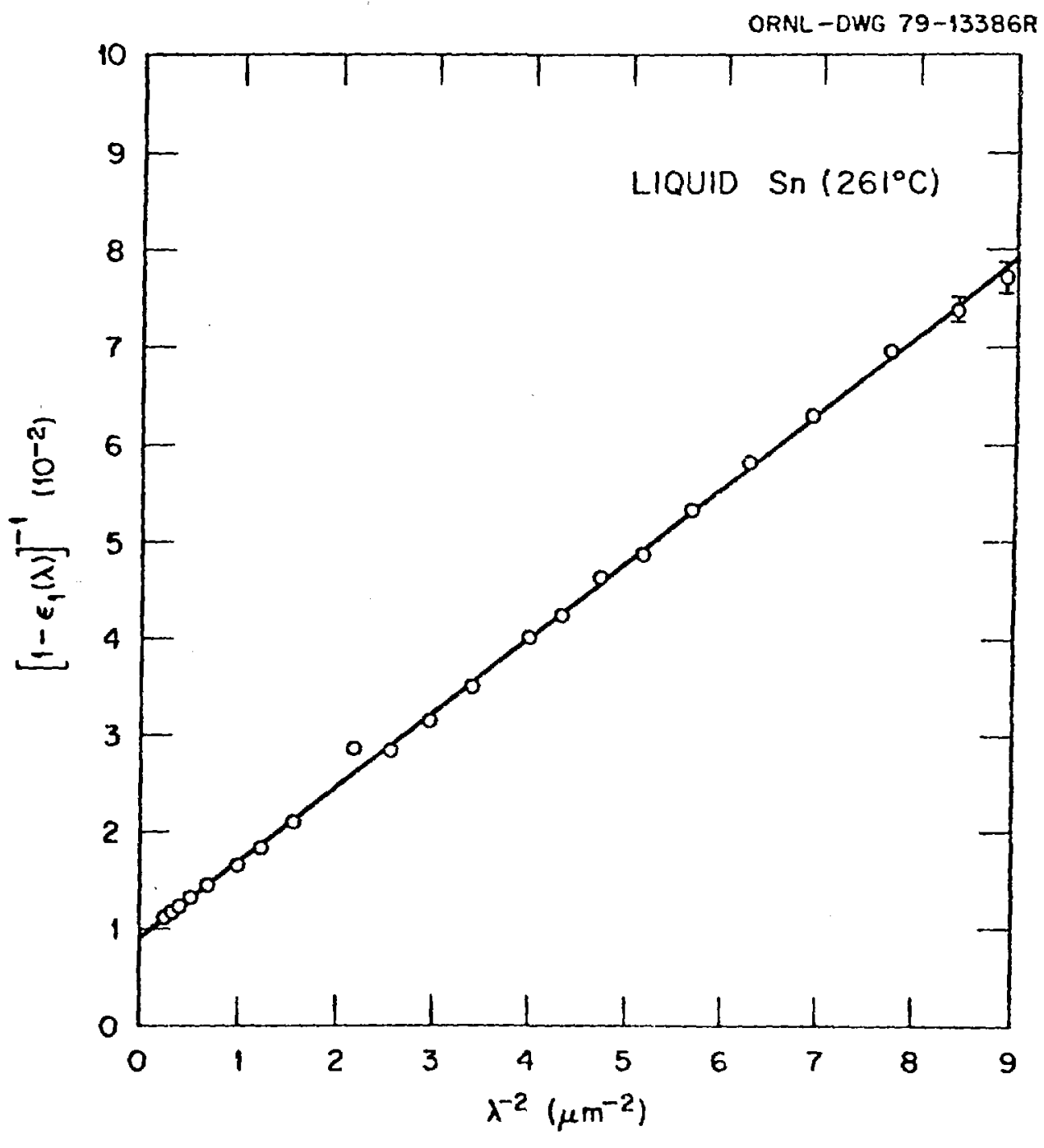

Figure 9 


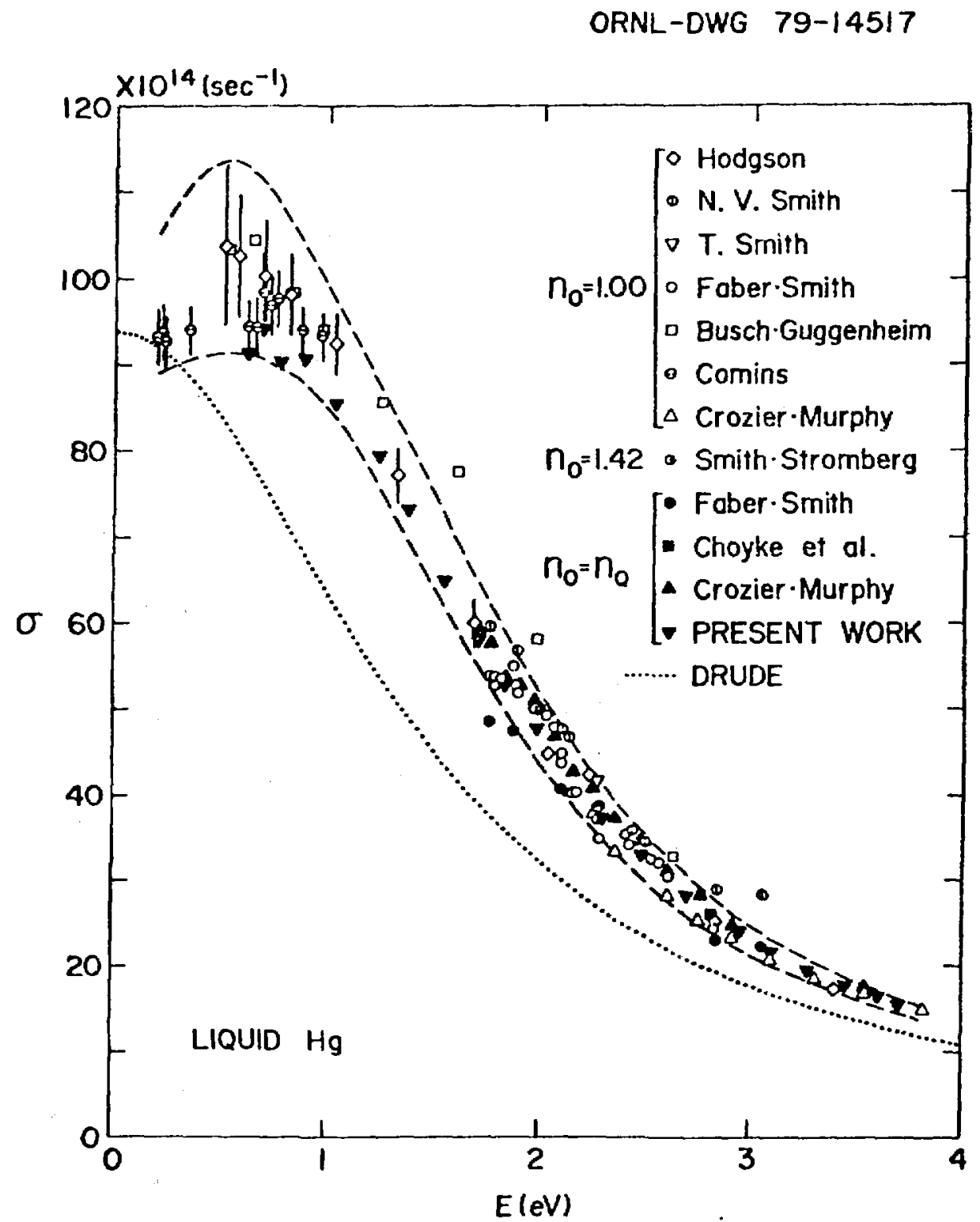

Figure 10 


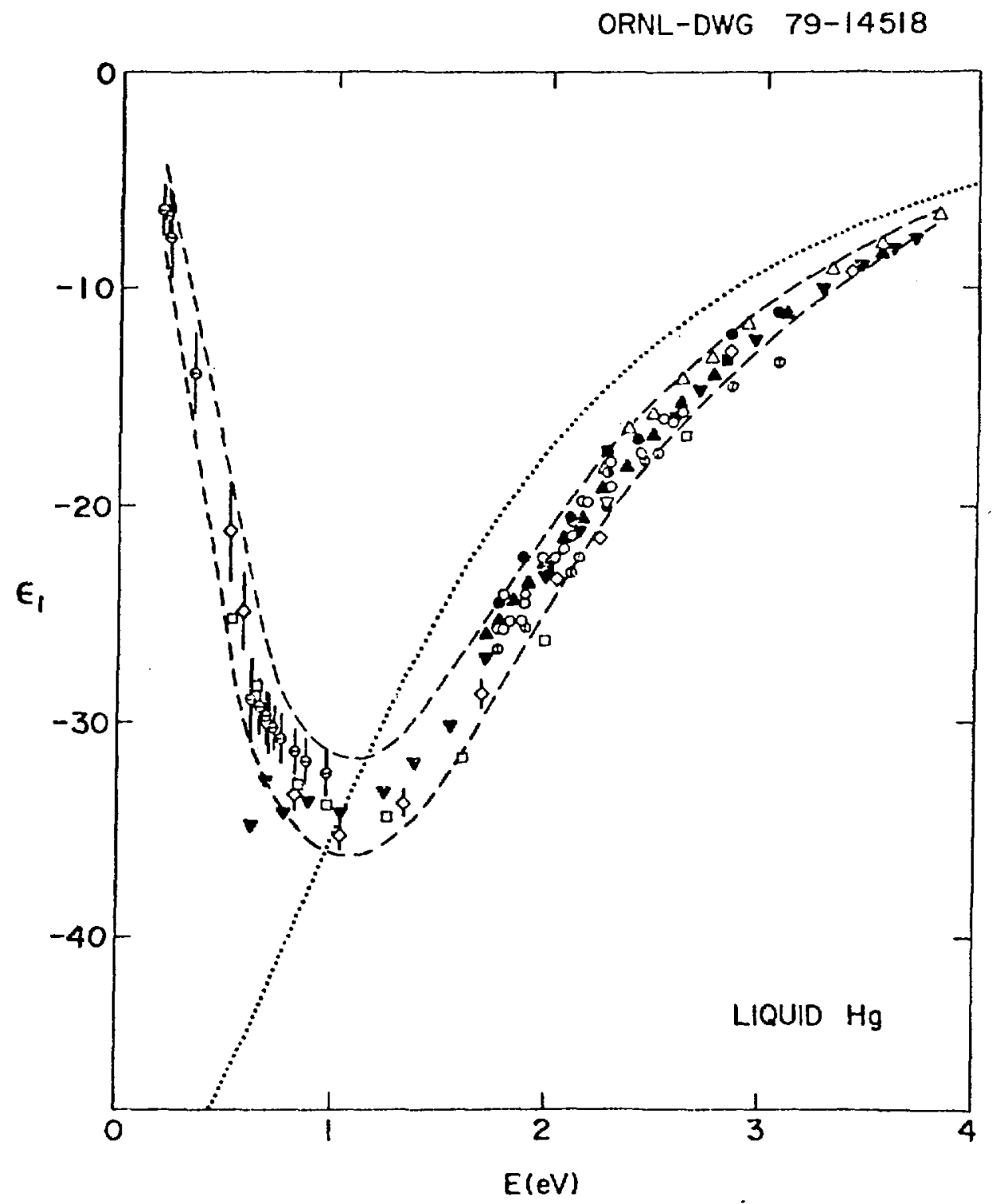

Figure 11 


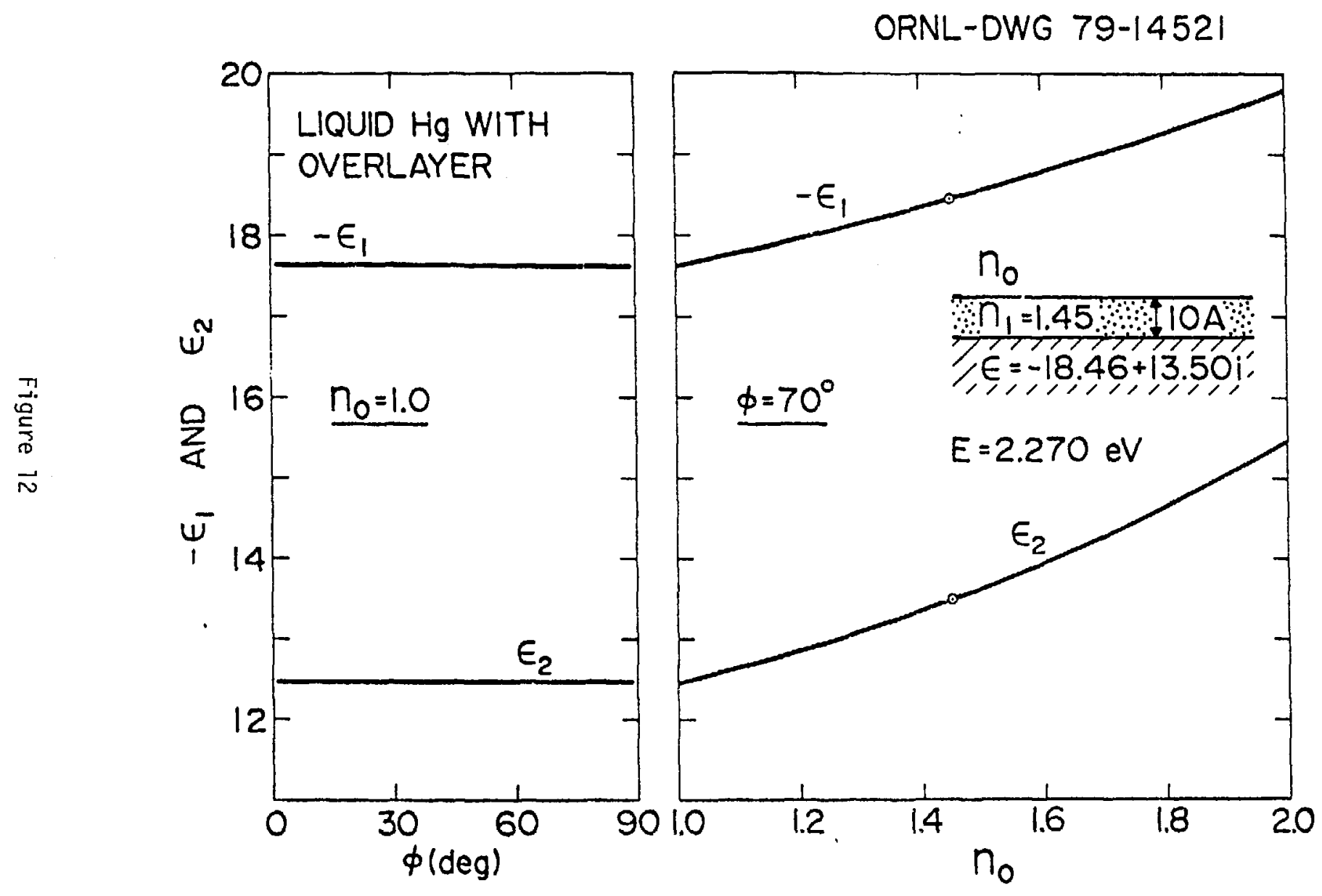



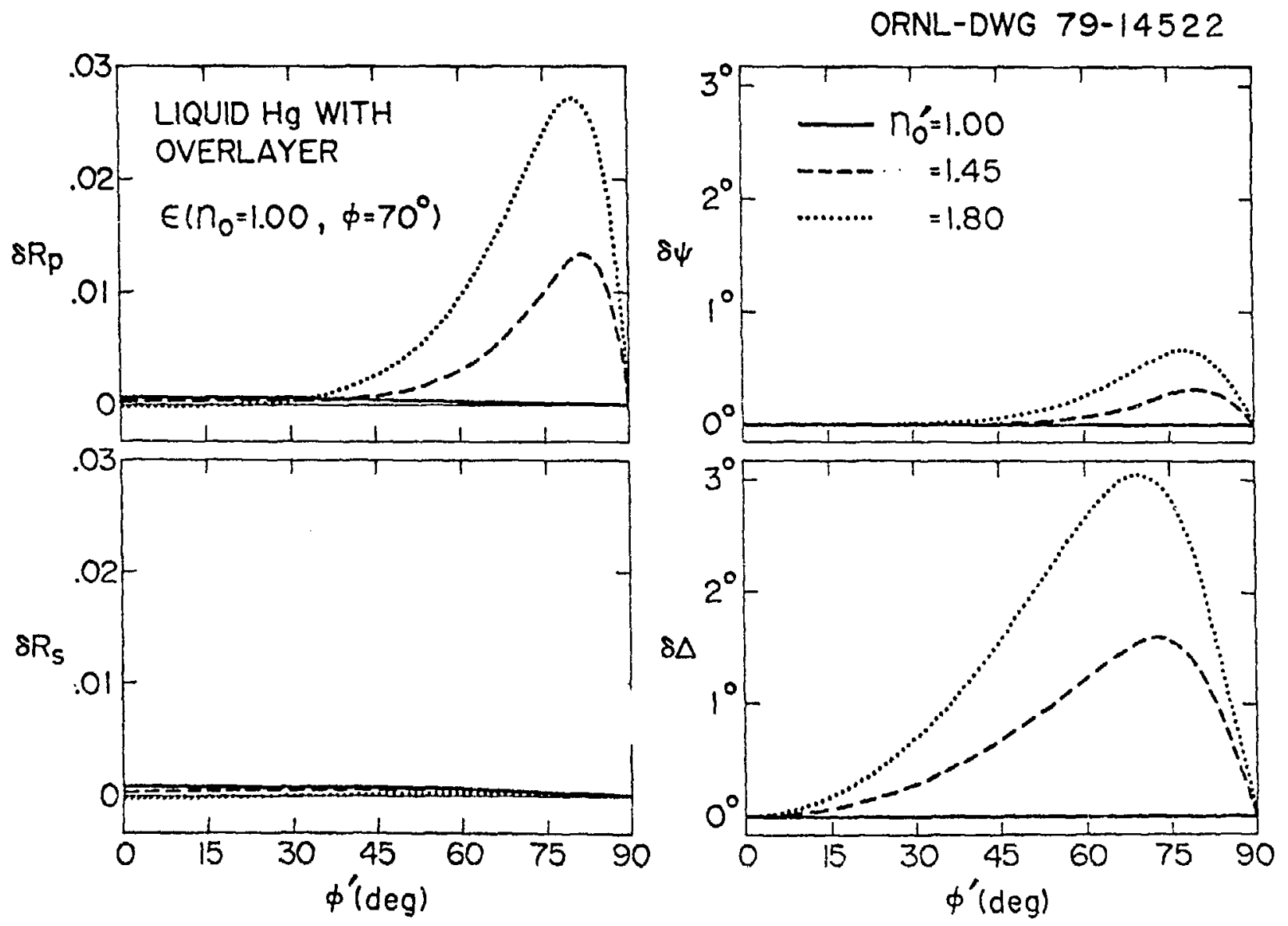

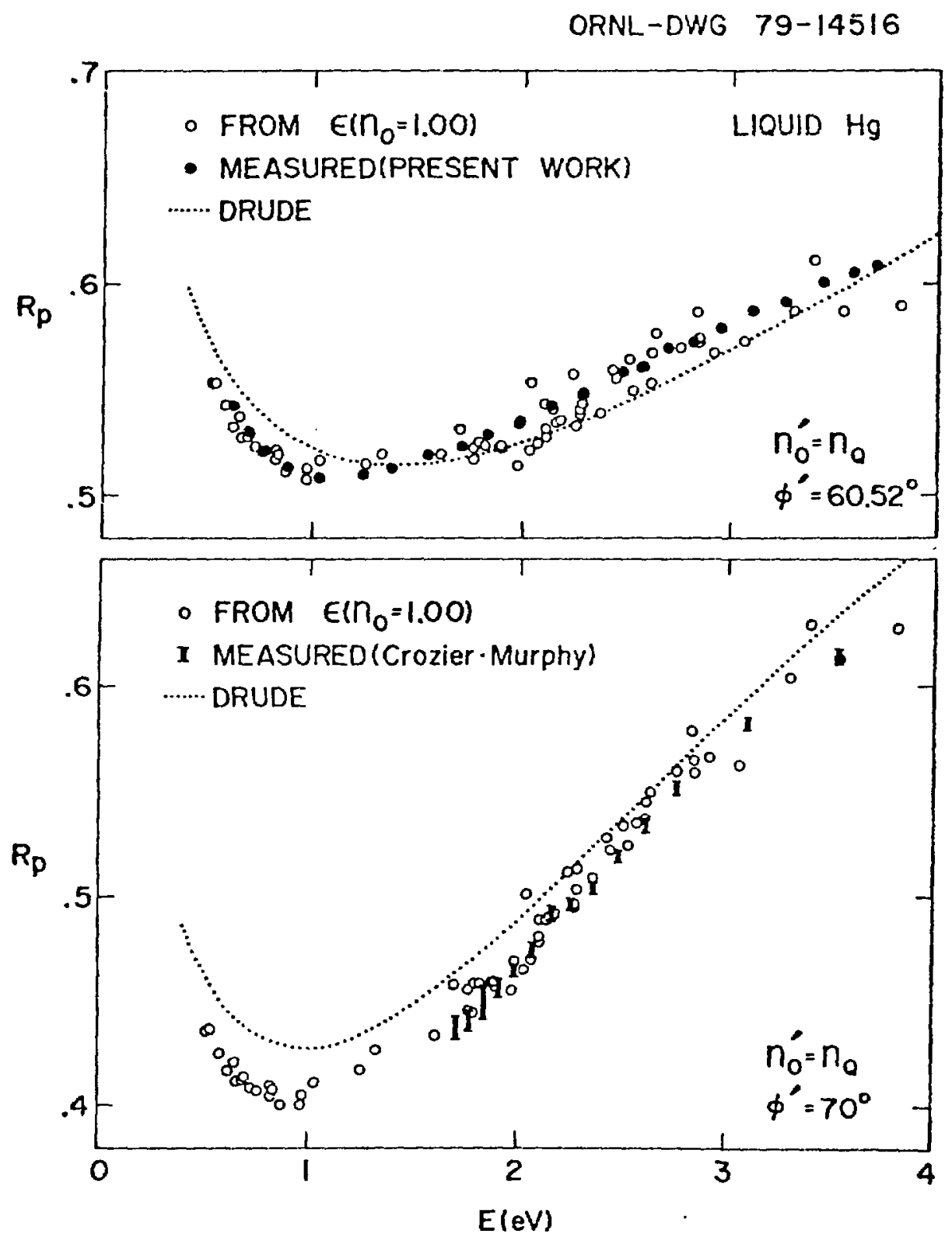

Figure 74 


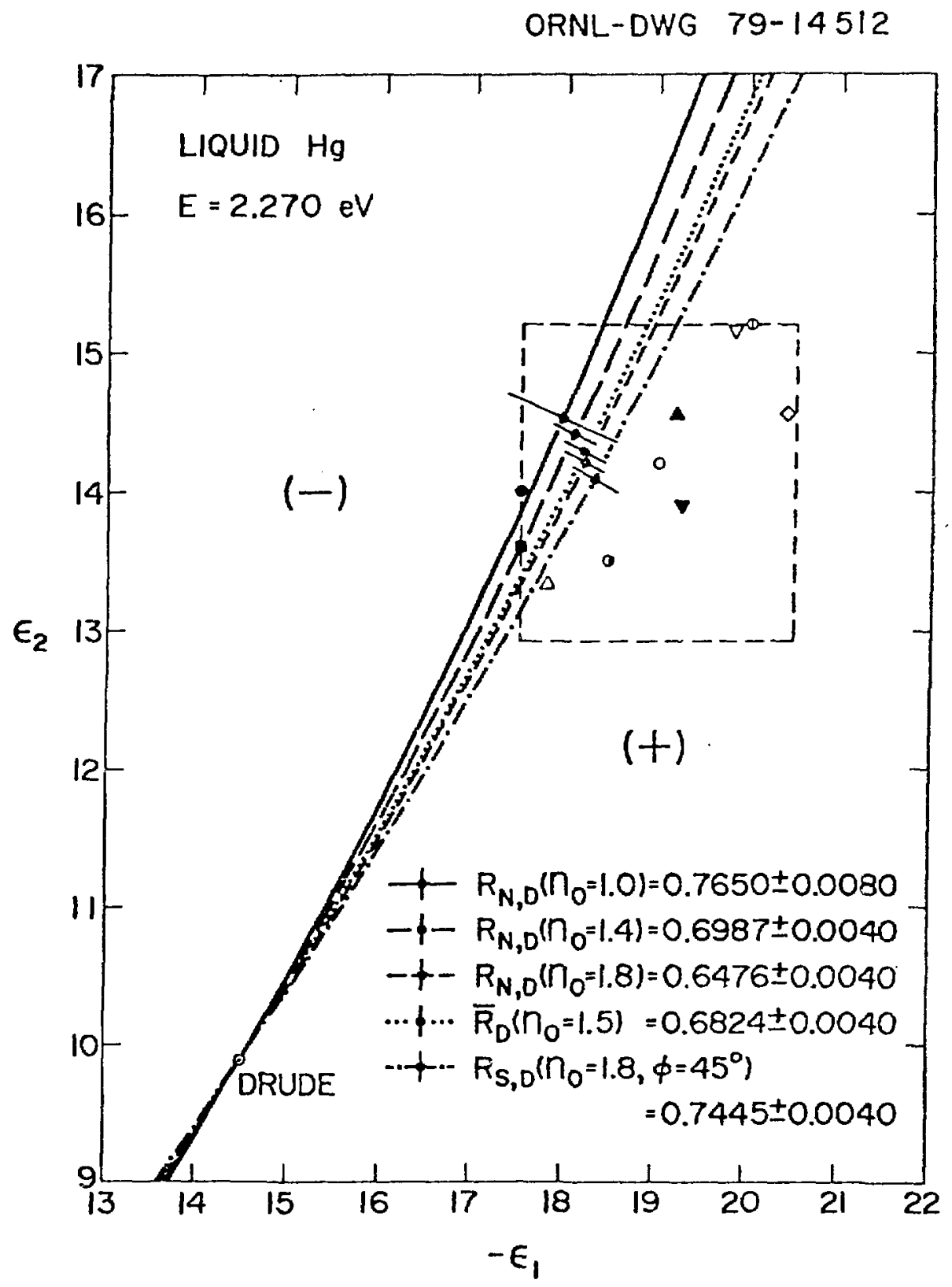

Figure 15 


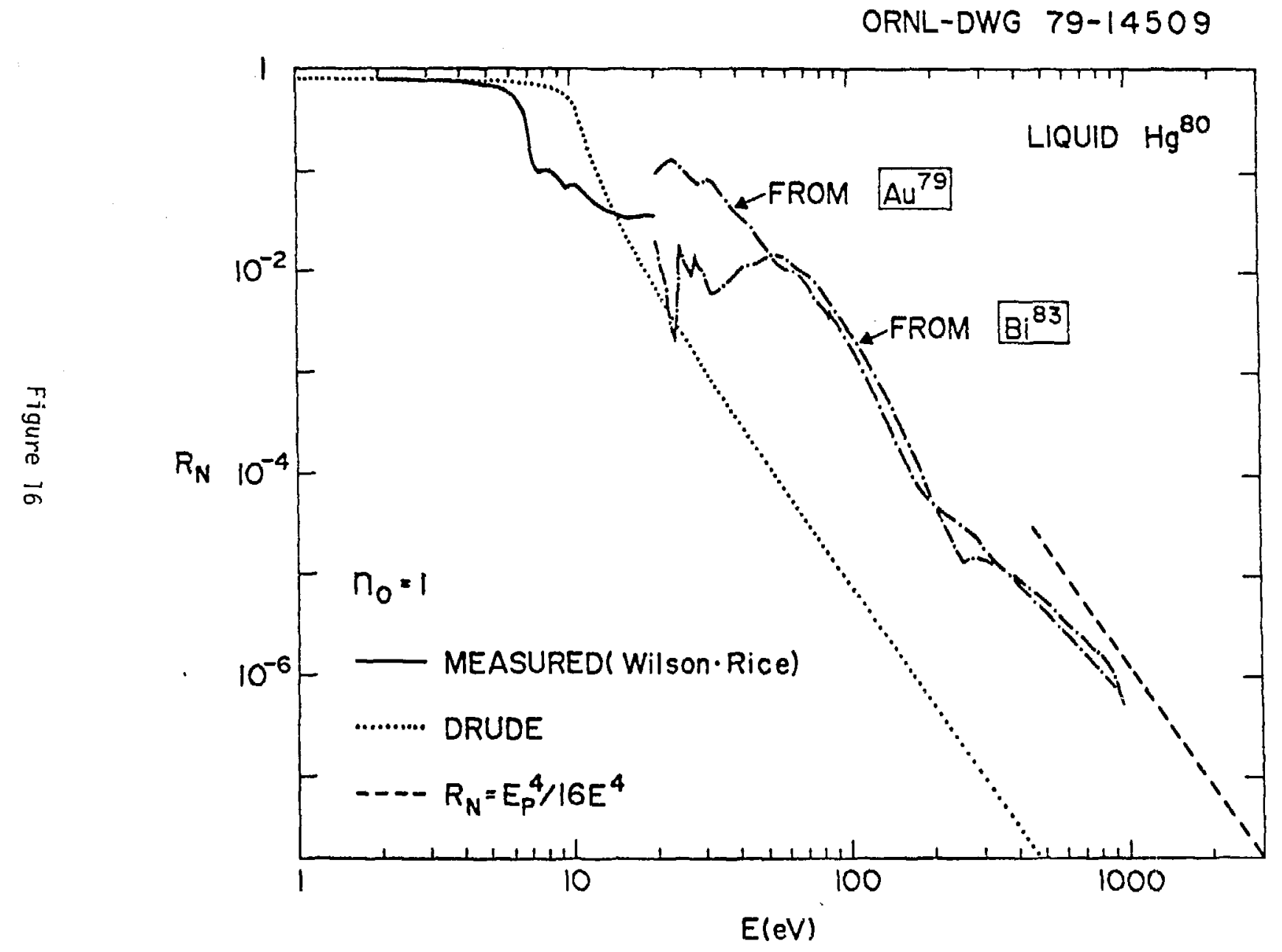




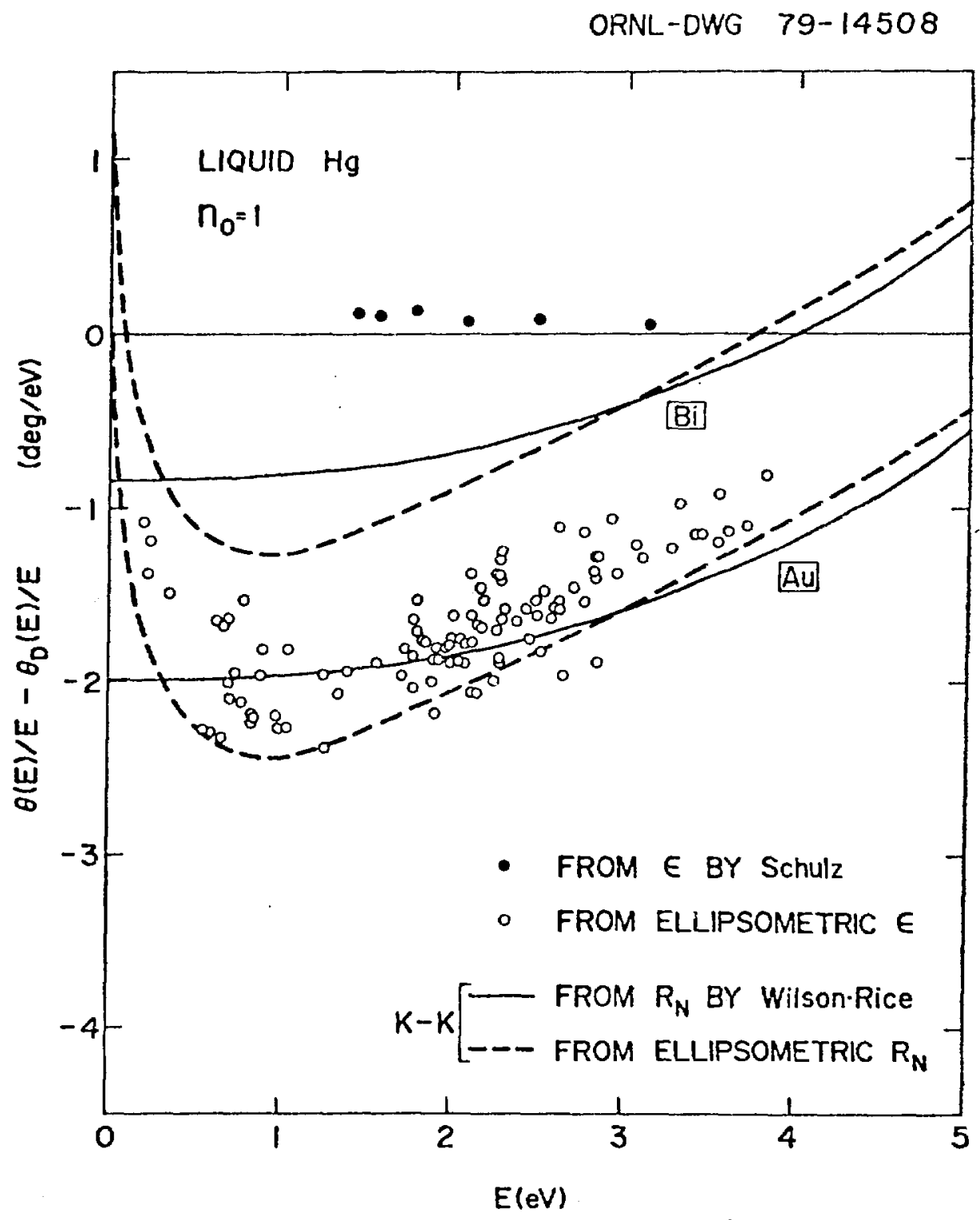

Figure 17 